\title{
Enhanced Sorption of Radionuclides by Defect-Rich Graphene Oxide
}

\author{
Nicolas Boulanger, ${ }^{\nabla}$ Anastasiia S. Kuzenkova, ${ }^{\nabla}$ Artem Iakunkov, Anna Yu. Romanchuk, \\ Alexander L. Trigub, Alexander V. Egorov, Stephen Bauters, Lucia Amidani, Marius Retegan, \\ Kristina O. Kvashnina, Stepan N. Kalmykov,* and Alexandr V. Talyzin*
}

Cite This: ACS Appl. Mater. Interfaces 2020, 12, 45122-45135

Read Online

ACCESS | 崩 Metrics \& More 回 Article Recommendations

Supporting Information

ABSTRACT: Extremely defect graphene oxide (dGO) is proposed as an advanced sorbent for treatment of radioactive waste and contaminated natural waters. dGO prepared using a modified Hummers oxidation procedure, starting from reduced graphene oxide ( $\mathrm{rGO}$ ) as a precursor, shows significantly higher sorption of U(VI), Am(III), and Eu(III) than standard graphene oxides (GOs). Earlier studies revealed the mechanism of radionuclide sorption related to defects in GO sheets. Therefore, explosive

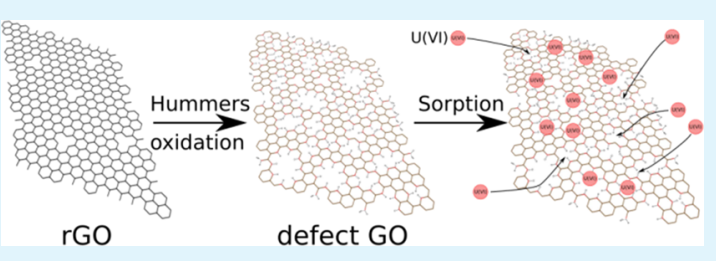
thermal exfoliation of graphite oxide was used to prepare rGO with a large number of defects and holes. Defects and holes are additionally introduced by Hummers oxidation of $\mathrm{rGO}$, thus providing an extremely defect-rich material. Analysis of characterization by XPS, TGA, and FTIR shows that dGO oxygen functionalization is predominantly related to defects, such as flake edges and edge atoms of holes, whereas standard GO exhibits oxygen functional groups mostly on the planar surface. The high abundance of defects in dGO results in a 15 -fold increase in sorption capacity of U(VI) compared to that in standard Hummers GO. The improved sorption capacity of dGO is related to abundant carboxylic group attached hole edge atoms of GO flakes as revealed by synchrotronbased extended X-ray absorption fine structure (EXAFS) and high-energy resolution fluorescence detected X-ray absorption near edge structure (HERFD-XANES) spectroscopy.

KEYWORDS: graphene oxide, sorption, radionuclides, uranium, defects

\section{INTRODUCTION}

Graphene oxide (GO) has been considered as an extremely promising material for treatment of radioactive waste and contaminated natural waters due to its high capacity in sorption of radionuclides. ${ }^{1-3}$ The processing of radioactive waste aims at converting it into forms convenient for long-term storage or permanent disposal. Many sorbent materials have been studied for the capture of radionuclides including zeolites, ${ }^{4,5}$ cement-based materials, ${ }^{6,7}$ clays, ${ }^{8-11}$ and carbon materials. $^{12-15} \mathrm{GO}$ as a true $2 \mathrm{D}$ material has a high theoretical specific surface area, which is important for efficient sorbent materials. ${ }^{16} \mathrm{GO}$ can be easily dispersed in water, exhibiting an experimental surface area determined by sorption of various molecules on the level of $700-800 \mathrm{~m}^{2} / \mathrm{g}^{16,17}$ with the theoretical surface value as high as $2600 \mathrm{~m}^{2} / \mathrm{g}$ for a single layered sheet. ${ }^{18}$ The high sorption ability of GO toward various cations of radionuclides and heavy metals has been demonstrated in several independent studies. ${ }^{2,3,19-25}$ The mechanism of radionuclides sorption by GO has been a subject of intense discussions over the past years. ${ }^{26}$ It is clear that understanding the chemistry behind the cation-GO interaction is extremely important for the design of a new generation of even more efficient sorbent materials. ${ }^{27}$ Tuning the properties of GO using different synthesis procedures and post preparation modifications provides possibilities for the further increase of GO sorption capacity.
Graphite oxides are synthesized by strong oxidation of graphite, most commonly using Hummers ${ }^{28}$ or Brodie $^{29}$ oxidation methods. Graphene oxide is easily produced using mild sonication of graphite oxides in water. Many variations of the Hummers procedure have also been proposed to make the dispersion of graphite oxides in water even more efficient. ${ }^{30}$ Properties of graphite oxides are significantly dependent on the method of preparation, e.g., thermal exfoliation temperatures, ${ }^{31,32}$ swelling, ${ }^{33-36}$ mechanical strength of individual flakes, ${ }^{37}$ sorption of polar solvents, ${ }^{38}$ and sorption capacity toward radionuclides. ${ }^{1}$ In fact, GOs are a family of materials with strong variation in both the degree of oxidation and relative numbers of various oxygen functional groups. ${ }^{39,40}$ Moreover, the composition of GO is affected by aging effects when stored in air. ${ }^{41-43}$ In general, for all types of GO, the planar surface of GO is functionalized mostly with epoxy and hydroxyl groups while the edges of GO flakes are predominantly terminated by carbonyls and carboxylic groups. ${ }^{44}$ Recent studies demonstrated that the defects in

Received: June 22, 2020

Accepted: September 9, 2020

Published: September 9, 2020 
GO sheets are not only always present and often abundant ${ }^{45,46}$ but also the defects (holes and vacancies) are responsible for some properties important for various applications. ${ }^{47-49}$

Our earlier study revealed that GO prepared using Hummers oxidation (HGO) and Tour variation of the Hummers method provides a higher capacity for sorption of radionuclides than $\mathrm{GO}$ synthesized by the Brodie method (BGO). ${ }^{1}$ BGO has fewer defects, higher relative amount of hydroxyl groups, and more homogeneous distribution of functional groups over its surface. ${ }^{33}$ HGO shows a relatively high percentage of carbonyl and carboxyl groups with a significant number of holes in the flakes and a strong disruption of the graphene structure. ${ }^{33}$ Therefore, enhanced sorption properties of HGO toward several radionuclides have been assigned to interactions with carboxylic groups on GO flakes. ${ }^{1}$ Preferential interaction of metal cations with carboxylic groups is in agreement with independent studies. ${ }^{27,50}$ Moreover, theoretical modeling, synchrotron radiation high-energy resolution fluorescence detected X-ray absorption near edge structure (HERFD-XANES), and extended X-ray absorption fine structure (EXAFS) data on local coordination of sorbed radionuclide atoms provided evidence for sorption predominantly inside the small holes and vacancy defects of GO sheets. ${ }^{1}$

It should be noted that standard synthesis of GO is usually optimized for the preparation of materials with a minimal number of defects, aiming mostly at converting it into defectfree graphene. ${ }^{37,51-53}$ Here, we propose to use extremely defect-rich GO for efficient sorption of radionuclides from aqueous solutions. Precursor HGO was explosively exfoliated to produce defect $\mathrm{rGO}^{54}$ The $\mathrm{rGO}$ was then oxidized using a mild Hummers procedure, which results in the formation of defect GO (dGO) with an abnormally high fraction of defects and functional groups with double bonded oxygen. Thanks to the very high number of defects and carboxylic groups, dGO shows strongly enhanced sorption of radionuclides.

\section{EXPERIMENTAL SECTION}

Starting Materials. Graphite oxide was purchased from Abalonyx (product 1.8, CAS 1034343-98-0). According to the provider, the GO material was prepared using a slightly modified Hummers method. The characterization by XPS, XRD, and FTIR confirmed that the material is standard HGO. The $\mathrm{C} / \mathrm{O}=1.99$ ratio was determined using XPS $(\mathrm{C} / \mathrm{O}=2.25$ not taking into account oxygen from sulfate groups). Trace amounts of nitrogen and $\mathrm{Si}$ were also detected as impurities.

According to our characterization, the GO confirms that all properties of this material are typical for standard HGO. Sodium nitrate was bought from Scharlab (CAS 7631-99-4), and sulfuric acid was purchased from Merck (95-97\% concentration, CAS 7664-93-9). Potassium permanganate (CAS 7722-64-7), hydrogen peroxide (30\% concentration, CAS 7722-84-1), and hydrochloric acid (37\% concentration, CAS 7647-01-0) were all purchased from VWR Chemicals. The methylene blue used for characterization was bought from Sigma-Aldrich (CAS 122965-43-9). The reference sample of porous templated carbon with a BET surface area of $\sim 2000 \mathrm{~m}^{2} / \mathrm{g}$ was purchased from ACS Material.

Synthesis of dGO. GO powder ( $4 \mathrm{~g}$ in one batch) was thermally exfoliated in air inside a large volume $(1-1.5 \mathrm{~L})$ container made of aluminum foil. The container is not sealed tightly to allow evolving gases to escape. The container was rapidly inserted into a furnace preheated to $240{ }^{\circ} \mathrm{C}$, annealed for $6 \mathrm{~min}$ with a closed furnace door, and removed from the furnace to provide rapid cooling. Rapid heating results in the explosion of GO powder and formation of rGO powder. ${ }^{55}$ This procedure for preparation of rGO provides maximal
BET surface area according to our earlier studies. ${ }^{54}$ Shortly, the maximal surface area or rGO is achieved using as rapid as possible heating at temperature $\sim 50^{\circ}$ above the main deflagration point determined using TGA $\left(\sim 190{ }^{\circ} \mathrm{C}\right)$. Using higher exfoliation temperatures, vacuum, or inert gas does not provide advantages for achieving higher surface area, but using a relatively large volume of the container is essential. ${ }^{54}$ Higher gas pressure (above ambient) has an adverse effect on exfoliation. ${ }^{55}$

rGO powder was used as a precursor for the oxidation using the standard Hummers procedure but with adjusted proportions between reagents.

For the synthesis of dGO, typically, $1 \mathrm{~g}$ of sodium nitrate was added into $40 \mathrm{~mL}$ of sulfuric acid while stirring. $1 \mathrm{~g}$ of rGO was then added, and the whole container was placed in an ice bath while stirring. Next, $1 \mathrm{~g}$ of potassium permanganate was slowly added to the mixture with frequent controls of the suspension temperature, keeping it below $\sim 20{ }^{\circ} \mathrm{C}$ as this is a very exothermic process. The example here cites the batch with a $\mathrm{rGO} / \mathrm{KMnO}_{4}$ ratio of $1: 1$. Note that the first attempt to synthesize dGO was performed using a 1:3 ratio and resulted in too strong oxidation (see the Results and Discussion section for details). When all the potassium permanganate was added, the suspension was preserved by stirring for a total time of $2 \mathrm{~h}$ counted after the first introduction of potassium permanganate.

The container was then placed in an oil bath heated at $30^{\circ} \mathrm{C}$ for 1 h. The container was then placed back into the ice bath and $40 \mathrm{~mL}$ of deionized water was very slowly added, as this too is a very exothermic process. The whole procedure occurred within a fume hood as strong fumes were generated during this step. Once the water was added to the suspension and the reaction seemed to stop, the container was placed back into the oil bath and maintained at $90{ }^{\circ} \mathrm{C}$ for $15 \mathrm{~min}$. The suspension was then taken out of the oil bath and placed at room temperature. $90 \mathrm{~mL}$ of $6 \%$ hydrogen peroxide was then added, and the mixture was left under stirring overnight at room temperature.

Finally, the mixture was rinsed by first washing it with $10 \%$ hydrochloric acid: the mixture was poured in centrifugation containers and mixed with the acid solution, shaken well, and centrifuged (Allegra 64R Centrifuge, Beckman Coulter) at $10000 \mathrm{rpm}$ for $10 \mathrm{~min}$. This washing process was repeated six times. Then, the remaining material was repeatedly washed with deionized water until the $\mathrm{pH}$ of the solution was around 4 to 5 . The mixture was each time shaken well and centrifuged at $20000 \mathrm{rpm}$ for $30 \mathrm{~min}$. The product was vacuum-filtered using a $1 \mu \mathrm{m}$ PTFE membrane (Omnipore, ref JAWP04700) and freeze-dried over a few days. The preparation of a batch using $9 \mathrm{~g}$ of $\mathrm{rGO}$ resulted in $8.6 \mathrm{~g}$ of dGO, which has a yield of 95.5\%.

Characterization. X-ray diffraction patterns for the powder samples were recorded using $\mathrm{Cu} \mathrm{K} \alpha$ radiation ( $\mathrm{X}^{\prime} \mathrm{Pert}^{3}$ Powder diffractometer by PANalytical). Fourier transform infrared (FTIR) spectra were recorded for powder samples using a Bruker IFS $66 \mathrm{v}$ spectrometer. The surface areas of the different materials were determined by recording nitrogen sorption isotherms using Autosorb iQ XR and Nova 1200 e surface area and pore size analyzers (Quantachrome) at liquid nitrogen temperature. The relative pressure $P / P_{0}$ for the BET plot was selected using a procedure optimized for microporous materials. Pore size volume and pore size distributions were determined using a slit-pore QSDFT equilibrium model.

Thermogravimetric analysis was performed using a Mettler Toledo TGA/DSC1 STAR ${ }^{\mathrm{e}}$ System. The measurements were performed from room temperature up to $800{ }^{\circ} \mathrm{C}$ at a heating rate of $5 \mathrm{~K} / \mathrm{min}$ under a nitrogen flow of $50 \mathrm{~mL} / \mathrm{min}$.

XPS spectra were recorded with an Axis Ultra DLD spectrometer (Kratos Analytical Limited, Great Britain) using $\mathrm{Al} \mathrm{K} \alpha$ radiation $(h \nu=$ $1486.6 \mathrm{eV}, 150 \mathrm{~W})$. The pass energy of the analyzer was $160 \mathrm{eV}$ for survey spectra and $40 \mathrm{eV}$ for high resolution scans. A Kratos charge neutralizer system was used, and the binding energy scale was adjusted with respect to the C1s line of aliphatic carbon set at 285.0 $\mathrm{eV}$. All spectra were processed with the Kratos software.

Sorption Measurements. Methylene blue (MB) sorption measurements were performed using a water solution of methylene 
blue and powders of tested materials using the UV-vis method. An aqueous solution of methylene blue was added to the solid powder material and stirred for 6 days. The concentration of $\mathrm{MB}$ and the proportion of solution to the amount of tested materials were selected to ensure that saturation sorption is achieved. Typically, $1 \mathrm{mg} / \mathrm{mL}$ concentration of methylene blue and $0.5 \mathrm{mg} / \mathrm{mL}$ tested material were used. The tested material was then removed by filtration, and the concentration of the remaining solution was determined by UV-vis measurements using a PerkinElmer Lambda 1050+ spectrometer. The $\mathrm{UV}$-vis absorbance of $\mathrm{MB}$ at $664 \mathrm{~nm}$ was recorded to determine the remaining $\mathrm{MB}$ concentration in solution. Calibration of UV-vis absorbance was performed for a set of $\mathrm{MB}$ solutions with known concentrations.

Sorption experiments with radionuclides were carried out in plastic vials as retention on the walls was found to be negligible. For the sorption experiments, an aliquot of the solution containing the radionuclides ${ }^{241} \mathrm{Am},{ }^{152} \mathrm{Eu}$, or ${ }^{233,232} \mathrm{U}$ was added to the $\mathrm{GO}$ suspension in $0.1 \mathrm{M} \mathrm{NaClO}_{4}$ (experiments with each radionuclide were performed separately). A mixture of ${ }^{233,232} \mathrm{U}$ and natural uranium was used in order to vary the uranium concentration for isotherm experiments. In the case of $\mathrm{Eu}$ isotherms, a solution of stable $\mathrm{Eu}(\mathrm{III})$ was used to make different total Eu concentrations. The concentration of the solid phase in most of the experiments was $0.7 \mathrm{~g} / \mathrm{L}$; however, it was $0.07 \mathrm{~g} / \mathrm{L}$ in the cases where the sorption on HGO was $\mathrm{pH}$ dependent. The $\mathrm{pH}$ value was measured using a combined glass $\mathrm{pH}$ electrode (InLab Expert Pro, Mettler Toledo) with an ionomer (SevenEasy pH S20-K, Mettler Toledo) and was adjusted via the addition of small amounts of dilute $\mathrm{HClO}_{4}$ or $\mathrm{NaOH}$. After equilibration, the GO suspension was centrifuged at $40000 \mathrm{~g}$ for 20 min (Allegra 64R, Beckman Coulter) to separate the solid phase from the solution. The sorption was calculated using the difference between the initial activity of the radionuclides and the activity measured in the solution after centrifugation. The activity of the radionuclides was measured using liquid-scintillation spectroscopy (Quantulus-1220, Perkin Elmer) and universal radiometric complex ORTEC DSPec50 (16013585).

For the purpose of EXAFS and FTIR characterization, samples equilibrated with only natural uranium were prepared. The concentration of the solid phase in this experiment was equal to $0.05 \mathrm{~g} / \mathrm{L}$ with the total concentration of $\mathrm{U}(\mathrm{VI})$ kept at $1 \times 10^{-5} \mathrm{M}$. The $\mathrm{pH}$ values were ca. 4.5-4.7.

EXAFS spectra were recorded at the Structural Materials Science beamline of the Kurchatov Synchrotron Radiation Source (Moscow, Russia) to analyze the $\mathrm{U}$ local atomic environment. A storage ring with an electron beam energy of $2.5 \mathrm{GeV}$ and a current of $80-100$ $\mathrm{mA}$ was used. The $\mathrm{X}$-ray beam was monochromatized using a $\mathrm{Si}(111)$ channel-cut monochromator, which provided an energy resolution of $\Delta E / E \approx 2 \times 10^{-4}$. Dumping of higher-energy harmonics was achieved by monochromator geometry distortion. The EXAFS spectrum of $\mathrm{Zr}$ foil was recorded for energy calibration. All experimental data were collected in transmission mode using ionization chambers filled with $\mathrm{N}_{2}$ and Ar. Simultaneous acquisition of EXAFS data for the sample and reference was achieved using three ionization chambers.

In this way, the monochromator energy calibration could be checked and corrected. At every energy point in the XANES region, the signal was integrated for $1 \mathrm{~s}$, while in the case of EXAFS, the integration time was set to $1 \mathrm{~s}$ at the beginning of the region and increased to $4 \mathrm{~s}$ at the end of the region. EXAFS data $\left(\chi_{\text {exp }}(k)\right)$ were analyzed using the IFEFFIT data analysis package. ${ }^{56}$ Standard procedures for the pre-edge subtraction and spline background removal were used for EXAFS data reduction. The radial pair distribution functions around the $U$ ions were obtained by the Fourier transformation (FT) of the $k^{2}$-weighted EXAFS functions $\chi_{\exp }(k)$ over the experimental ranges. Experimental spectra were fitted in R-space within the range of $1.2-4.2 \AA$. For the refined interatomic distances $\left(R_{\mathrm{i}}\right)$, the statistical error is $0.01-0.02 \AA$ for the first coordination sphere.

ATR-FTIR spectra were recorded using a PLATINUM, a single reflection horizontal ATR accessory from Bruker Technologies, equipped with a diamond crystal. Unground small species of the samples were analyzed at room temperature. For each sample, 256 scans were recorded under vacuum in the MIR region (4000-400 $\mathrm{cm}^{-1}$ ) with a resolution of $4 \mathrm{~cm}^{-1}$.

The $\mathrm{U} \mathrm{M}_{4}$ edge HERFD-XANES spectra were recorded at the ACT station of the CAT-ACT beamline of the KARA (Karlsruhe Research Accelerator) facility in Karlsruhe, Germany. ${ }^{57,58}$ A storage ring with an electron beam energy of $2.5 \mathrm{GeV}$ and a current of $70-$ $100 \mathrm{~mA}$ was used. The incoming X-ray beam was monochromatized by a $\mathrm{Si}(111)$ double crystal monochromator and slitted down to 500 $\times 500 \mu \mathrm{m}$ at the sample position. The sample environment, including the entire Johann-type emission spectrometer, is contained within a He box to improve X-ray detectability and ensure sample stability. The samples were contained in the standard CAT-ACT pellet sample holder, using a double Kapton confinement of 8 and $12.5 \mu \mathrm{m}$ thickness on the front side. To obtain the best possible resolution on the 5-crystal emission spectrometer, only 1 masked Si (220) crystal with a bending radius of $1 \mathrm{~m}$ was set at the $\mathrm{U} \mathrm{M} \beta$ emission energy $(3339.8 \mathrm{eV})$. Several continuous scan mode spectra were averaged to obtain the final results.

\section{RESULTS AND DISCUSSION}

Synthesis and Characterization of dGO. Extremely defect-rich GO was synthesized in our study using the Hummers oxidation procedure applied to the rGO precursor prepared using explosive thermal exfoliation of standard HGO. ${ }^{54}$ Defects and holes in GO sheets are formed already during the synthesis by the Hummers procedure. ${ }^{45}$ Therefore, we suggested that reduced GO will keep these holes and after re-oxidation of $\mathrm{rGO}$ the number of holes will further increase.

It is well known that the result of Hummers oxidation depends very strongly on the type of precursor. ${ }^{17,31}$ Well crystalline graphite is typically used as a precursor for Hummers oxidation aimed at preparation of GO with the lowest possible amount of defects. The idea of this study was to use rather defected $\mathrm{rGO}$ as a precursor for Hummers oxidation in order to prepare extremely defected GO.

Rapid thermal exfoliation of graphite oxide results in the formation of a few-layered rGO material with specific surface area (SSA) on the level of $300-600 \mathrm{~m}^{2} / \mathrm{g}$ depending on how rapid the temperature is increased. ${ }^{54}$ In contrast to chemical reduction of $\mathrm{GO}$, which preserves the graphene skeleton relatively intact, explosive thermal exfoliation provides larger numbers of defects in rGO sheets. ${ }^{59,60}$ It was argued already back in the 60s that holes and vacancies are formed due to rapid formation of gaseous carbon oxides and explosion driven by the buildup of gas pressure inside the graphite oxide interlayers. ${ }^{17}$ It is well known that rapid thermal deflagration of GO results in evolving $\mathrm{CO}$ and $\mathrm{CO}_{2}$ gases. ${ }^{61}$ Therefore, carbon atoms are removed from the graphene skeleton in the process of thermal deoxygenation providing formation of vacancy defects and holes. The defects also prevent restacking of rGO sheets providing high BET surface area values. Rapid heating and stronger explosion result in GO with maximal surface area and maximal number of defects. ${ }^{54,55,60}$

Oxidation of the defect rGO material was anticipated to result in a GO material with many holes and vacancies per unit area, thus increasing the number of edge carbons typically functionalized with carbonyl and carboxylic groups. Therefore, the formation of holes and vacancies occurs in the process of precursor GO synthesis, in the process of thermal explosive exfoliation, and in the process of Hummers oxidation of explosively produced rGO. The carboxylic groups inside the holes of dGO could be expected to serve as sorption sites for the removal of radioactive waste from aqueous solutions (Figure 1). 


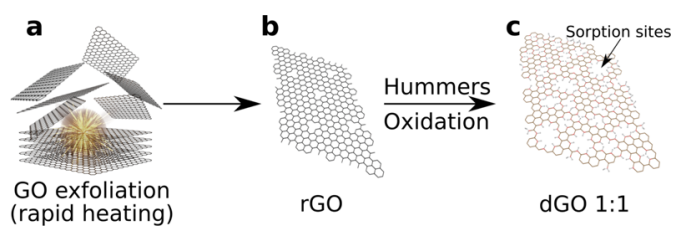

Figure 1. Scheme of defect GO preparation: (a) explosive thermal exfoliation of $\mathrm{HGO}$ to produce $\mathrm{rGO}$, (b) using defect rGO for Hummers oxidation, and (c) formation of holey dGO with sorption sites on the holes edges.

The first attempt to oxidize rGO was performed using a Hummers procedure very similar to the one used for the preparation of standard $\mathrm{HGO}$, with a $\mathrm{rGO} / \mathrm{KMnO}_{4}$ proportion of $1: 3$. However, when few-layered and strongly defected rGO was used instead of graphite powder, the oxidation proceeded too far. Most of the rGO powder was converted into dark brown solution with only very little dGO powder. The brown solution did not yield any precipitate even after using ultracentrifugation $(\sim 60000 \mathrm{~g}$ for $4 \mathrm{~h}$ and passed through the finest filters (10000 a.u.)). Some powdered material could be obtained from this solution by evaporation or using chemical precipitation by adding $10 \% \mathrm{NH}_{4} \mathrm{Cl}$ solution. These are not convenient methods for practical sorbent applications. It is obvious that extremely strong oxidation resulted in the breaking up of GO sheets into smaller and smaller fragments until some water soluble molecules were formed.

The difficulty in removing the dGO material from solution motivated us to use a milder oxidation procedure. The initial $\mathrm{rGO} / \mathrm{KMnO}_{4}$ of $1: 3$ ratio was decreased to $1: 1$. This synthesis provided a sufficient amount of powdered material easily precipitated by centrifugation. In the following discussions, the two dGO materials will be named dGO (1:1) and dGO (1:3) according to the proportion between the carbon precursor and potassium permanganate. Most of the experiments were performed on dGO (1:1).

Characterization of dGO was performed using XRD, Raman, and FTIR spectroscopies, analysis of $\mathrm{N}_{2}$ sorption isotherms, and XPS. Analysis of data, collected using a combination of these methods, demonstrated that the material produced by reoxidation of rGO is extremely defect-rich GO.

Both dGO materials appeared to be amorphous in XRD tests yielding no (001) reflection, typical for standard HGO (Figure 2). The absence of a well aligned layered structure is expected for strongly defected GO sheets due to the large number of vacancies and holes disrupting the typical planar shape.

The chemical composition and quantitative estimation for the relative number of in-plane and edge carbon atoms can be evaluated using XPS data (Figure 3).

The most common interpretation of XPS spectra assigns C1s peaks of GO as follows: $285.0 \mathrm{eV}$ to $\mathrm{C}-\mathrm{C}$ and $\mathrm{C}-\mathrm{OH}$ groups, $286.6 \mathrm{eV}$ to epoxy $\mathrm{C}-\mathrm{O}-\mathrm{C}$ groups, and $288.9 \mathrm{eV}$ to double bonded $\mathrm{C}=\mathrm{O}$, which includes carboxyls and carbonyls. Note that the C1s component due to hydroxyl groups is not resolved from $\mathrm{C}-\mathrm{C}$ in XPS spectra, which makes overall quantitative analysis using only $\mathrm{C} 1 \mathrm{~s}$ deconvolution not reliable. However, it can confidently be concluded that dGO shows a strongly increased \% of carboxyl/carbonyl functional groups compared to standard HGO. Formation of double bonded $\mathrm{C}=\mathrm{O}$ is possible only on the edges of $\mathrm{GO}$ flakes or in vacancy defects.

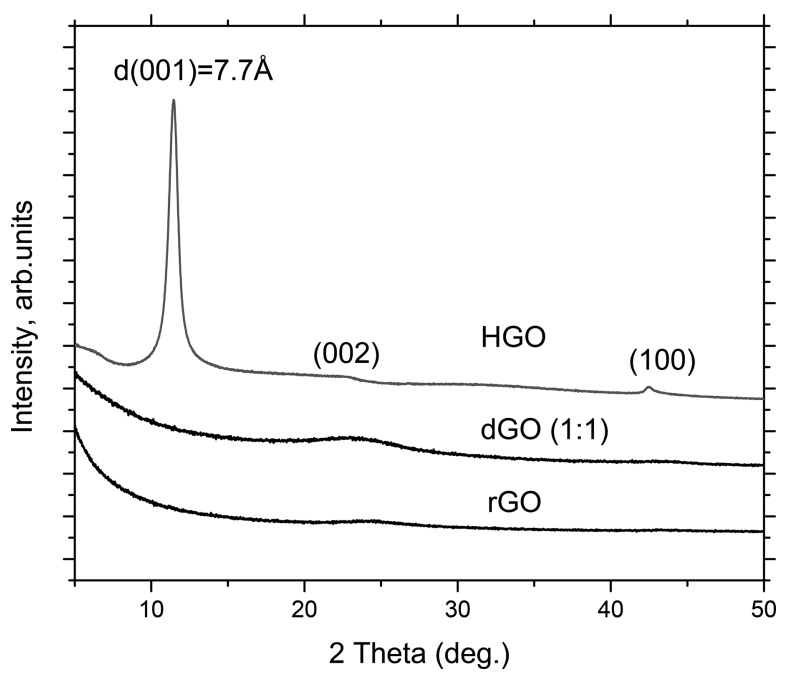

Figure 2. XRD patterns of HGO (intensity divided by 10), dGO (1:1), and precursor rGO. The pattern of HGO was scaled down in intensity.

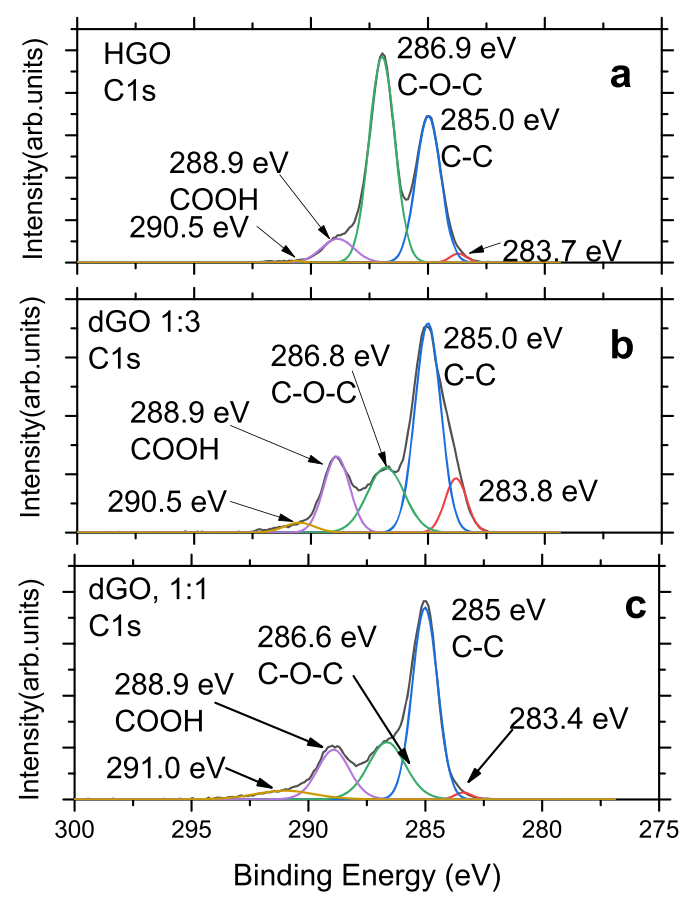

Figure 3. C1s XPS spectra of (a) standard HGO, (b) dGO (1:3), and (c) dGO (1:1).

Formation of a large number of holes in GO flakes is expected also to result in a smaller relative number of functional groups located on planes. Indeed, dGO shows a much lower intensity of the $\mathrm{C} 1 \mathrm{~s}$ component due to epoxy groups, which are attached to the planar surface of flakes.

The significant difference between standard HGO and dGO is also confirmed by analysis of the O1s part of XPS spectra (Figure 4). Standard HGO shows mostly one kind of oxygen with a major $\mathrm{O} 1 \mathrm{~s}$ peak at $532.7 \mathrm{eV}$, which includes all oxygen attached to planes and a rather minor component at $531.2 \mathrm{eV}$ due to edge groups. The O1s spectrum of dGO shows two peaks with similar intensity, indicating that almost $50 \%$ of oxygen is in carboxyl and carbonyl groups. 

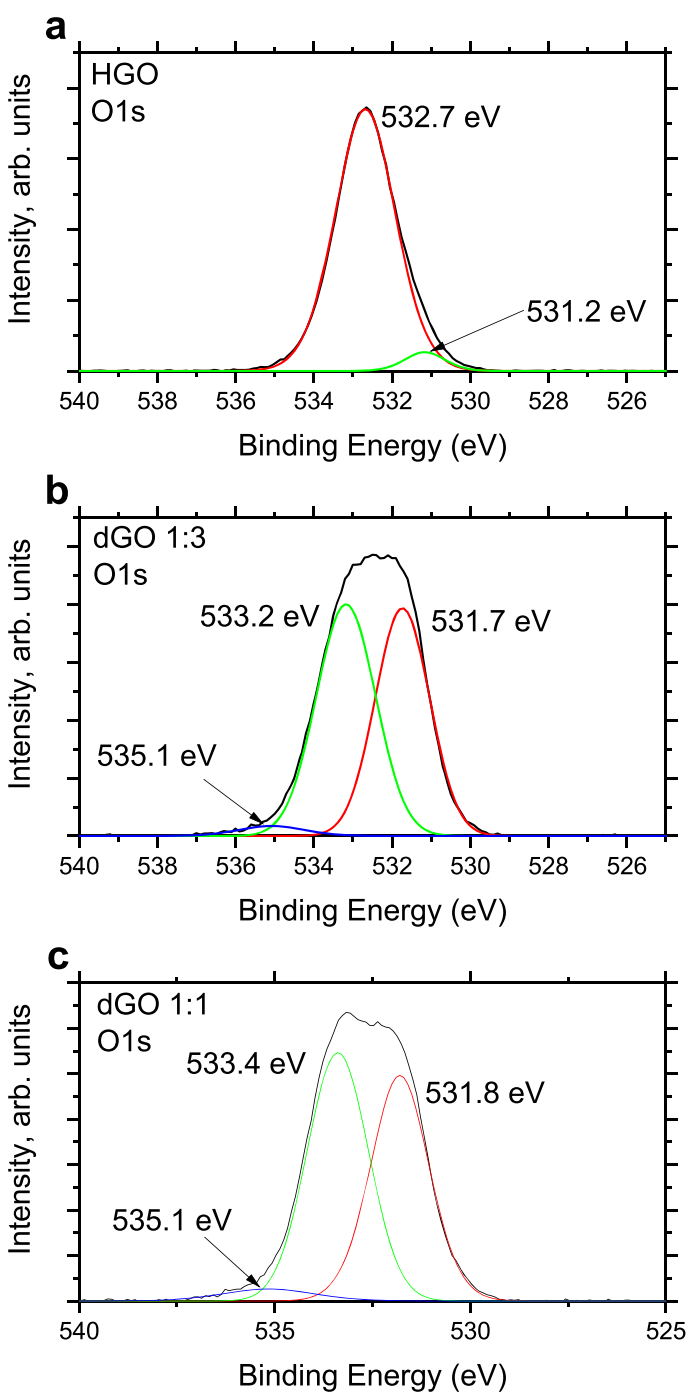

Figure 4. O1s XPS spectra of (a) standard HGO, (b) dGO (1:3), and (c) dGO (1:1).

The very high percentage of edge carbons detected by XPS in dGO can be explained either by the very small size of flakes or by a large number of holes present. However, XPS does not allow two kinds of edges to be distinguished: inner edges of holes and outer flake edges.

The possible relative amount of these two types of edge carbon atoms can be estimated using a simple model (Figure 5). Assuming the smallest graphene particle to be the size and shape of a coronene molecule, a set of progressively larger graphene particles of similar shape diameter (see the inset in Figure 5) was considered and the percentage of edge atoms was plotted as a function of the particle. It is clear that particles of irregular shape provide different proportions in the numbers of edge atoms to plane atoms. However, the plot shown in Figure 5 anyway provides a rough estimation for the analysis of XPS data. The precursor GO material with $5 \%$ of edge atoms placed only on the flake edges corresponds in this model to a particle size only $20 \mathrm{~nm}$ in diameter. However, the particle size evaluated for the standard HGO material using AFM and SEM is on average about $300-500 \mathrm{~nm}$, with some particles in micrometer size. ${ }^{43}$ For dGO ( $\sim 11 \%$ of edge carbons), the model provides diameters of particles as small as $8 \mathrm{~nm}$.

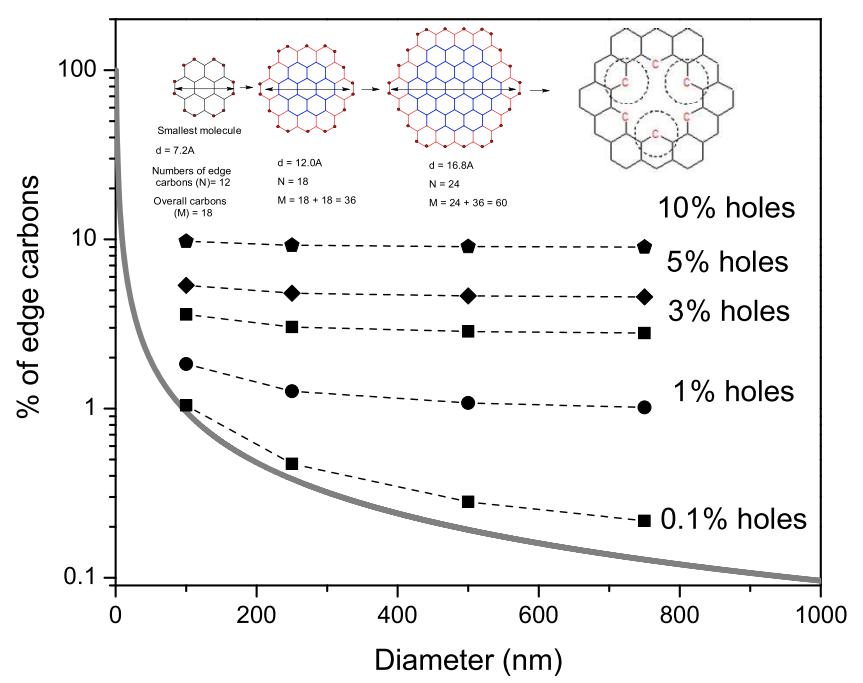

Figure 5. Percentage of edge carbon atoms as a function of "coronene-shaped" graphene particle size. Square symbols connected by the dashed line illustrate the $\%$ of edge atoms when small holes are added to graphene particles. Holes with a size equal to one removed hexagon are considered for this plot (see the inset). The number of holes is expressed by the percent of the total area occupied by holes $(0.1-10 \%$ of total area).

However, the contribution from carbon atoms located on the inner edges of holes needs also to be considered.

The relatively large percentage of carboxyl and carbonyl groups in HGO can be explained by assuming the existence of holes and point defects. It is clear that holes in GO sheets might have a variety of shapes and sizes, all affecting the proportion of edge atoms. As a simplified example, we considered a hole in the graphene sheet formed by removing 6 carbon atoms (one hexagon). This hole has 6 edge atoms and is large enough to accommodate 3 carboxylic and 3 carbonyl groups. The overall amount of holes is expressed by the percent of the area in holes relative to the total particle area. Assuming a realistic area of holes to be in the range of 1$10 \%$, several points were added to the plot in Figure 5. Remarkably, a high \% of edge carbon atoms inside the holes (e.g., $\sim 10 \%$ similar to the experimental value for dGO) can be achieved according to the model for any GO particle size assuming only $10 \%$ of total area to be in holes.

It should be noted that the direct experimental estimation of area covered by holes in GO sheets is very difficult even using high-resolution TEM. GO is very sensitive to electron beam radiation and degrades immediately unless some very soft and very technically demanding conditions are implemented. Several studies where HRTEM of "graphene oxide" was presented showed instead almost completely reduced graphene sheets. The best estimate using HRTEM of standard HGO has about $2 \%$ of area in holes with diameter less than $5 \mathrm{~nm}^{45}$ Using Figure 5, we can estimate that $2 \%$ of the area corresponds to approximately $4-5 \%$ of edge atoms for almost any reasonable GO flake size. This value of $4-5 \%$ edge atoms is in very good agreement with experimental XPS data for standard HGO (Figure 2 and Table 1).

The size of dGO (1:1) particles was analyzed using AFM and SEM (Figure 6). Most of the particles were found to be in the range of $30-100 \mathrm{~nm}$, but few particles of larger size were also detected. Note that the particles tend to aggregate when 
Table 1. XPS C1s Data for HGO and dGO Samples ${ }^{a}$

\begin{tabular}{|c|c|c|c|c|c|c|}
\hline sample & $\mathrm{C} / \mathrm{O}$ & $283.4-283.6 \mathrm{eV}$ & $285.0 \mathrm{eV}(\mathrm{C}-\mathrm{C})$ & $286.6-286.8 \mathrm{eV}(\mathrm{C}-\mathrm{O})$ & $288.9 \mathrm{eV}(\mathrm{C}=\mathrm{O})$ & $290.5-291.0 \mathrm{eV}$ \\
\hline precursor GO & $1.95(2.2)^{*}$ & 0.98 & 23.78 & 34.68 & 5.07 & 0.13 \\
\hline dGO 1:1 & 2.7 & 0.93 & 37.51 & 16.79 & 12.49 & 4.11 \\
\hline dGO $1: 3$ & 2.1 & 6.83 & 30.99 & 11.88 & 11.39 & 1.76 \\
\hline
\end{tabular}

${ }^{a} \mathrm{C} / \mathrm{O}$ ratio and \% of area (at. \%) are based on deconvolution of spectra shown in Figure 3 . The value $\mathrm{C} / \mathrm{O}=2.2$ is calculated excluding oxygen from sulfate groups in standard HGO. No sulfur was detected in the XPS spectrum of dGO. The C=O peak includes contributions from carboxyl and carbonyl groups.

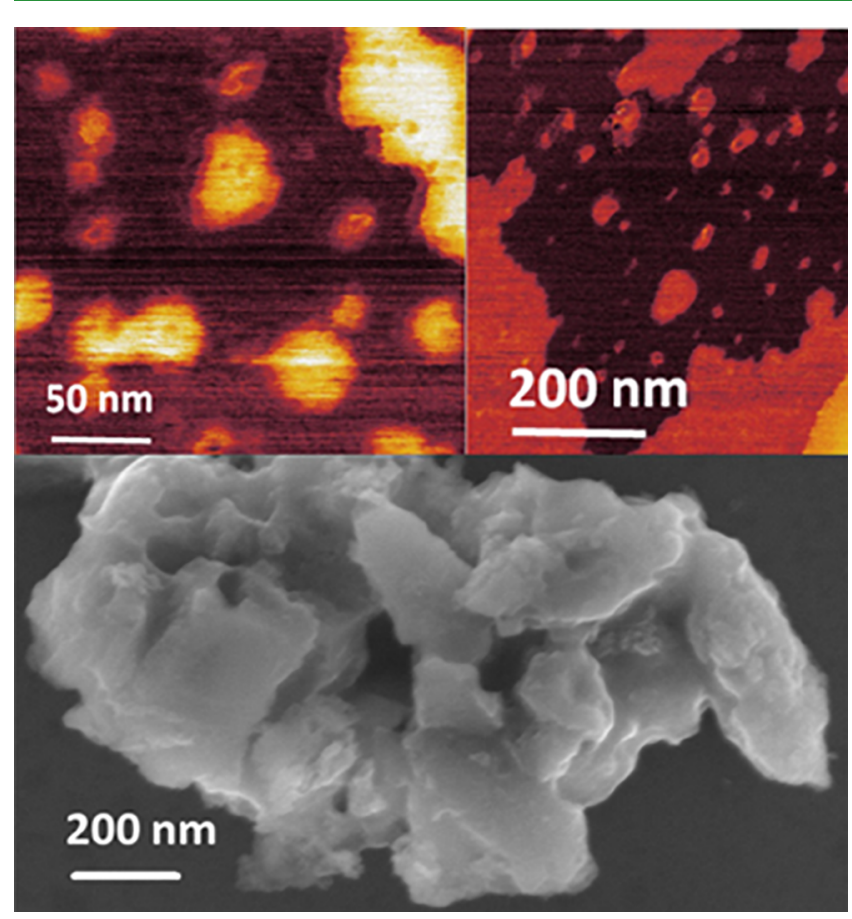

Figure 6. AFM (phase image) (top) and SEM (bottom) images of dGO (1:1).

precipitated and it is not always possible to distinguish individual particles from aggregates in SEM.
The oxidation degree of dGO determined by XPS is also confirmed using TGA data. The total weight loss over a temperature interval from ambient up to $700{ }^{\circ} \mathrm{C}$ due to heating under nitrogen is about 55\%, similar to standard HGO ( $\sim 60 \%)$. However, standard HGO shows most of the weight loss in two sharp steps, firstly due to water desorption below $100{ }^{\circ} \mathrm{C}(\sim 8 \%$, region I) and secondly due to the removal of oxygen functional groups from the planar surface around 150$240{ }^{\circ} \mathrm{C}(\sim 30 \%$, region II $)$.

Slow weight loss at temperatures above $\sim 370{ }^{\circ} \mathrm{C}$ (region III) is usually assigned to the removal of other oxygen groups (mostly carboxylic and carbonyls) attached to edges.

The TGA trace of dGO shows both steps typical for standard GO, but only about $12 \%$ of weight is lost due to the removal of oxygen from the planar surface. The main weight loss $(\sim 24 \%)$ in dGO occurs gradually at temperatures above $\sim 350{ }^{\circ} \mathrm{C}$ (Figure 7 ). Therefore, TGA data are in agreement with the suggested extremely defected structure of dGO with a very large number of edge atoms and a decreased area of the planar sheet surface. Thermal exfoliation of dGO results in the formation of reduced dGO (rdGO) but without a significant increase of BET surface area. HGO typically shows BET $\left(\mathrm{N}_{2}\right)$ surface area about $10-30 \mathrm{~m}^{2} / \mathrm{g}$, which increases up to $300-$ $500 \mathrm{~m}^{2} / \mathrm{g}$ after rapid thermal exfoliation. ${ }^{54,55}$ The BET surface area of $\mathrm{dGO}$ remains almost unchanged after rapid thermal exfoliation, thus providing additional arguments for the holey structure of dGO (Figure S1). The gases formed in a process of thermal deoxygenation are likely to escape easily through the defects of dGO sheets. As a result, the gas pressure buildup,
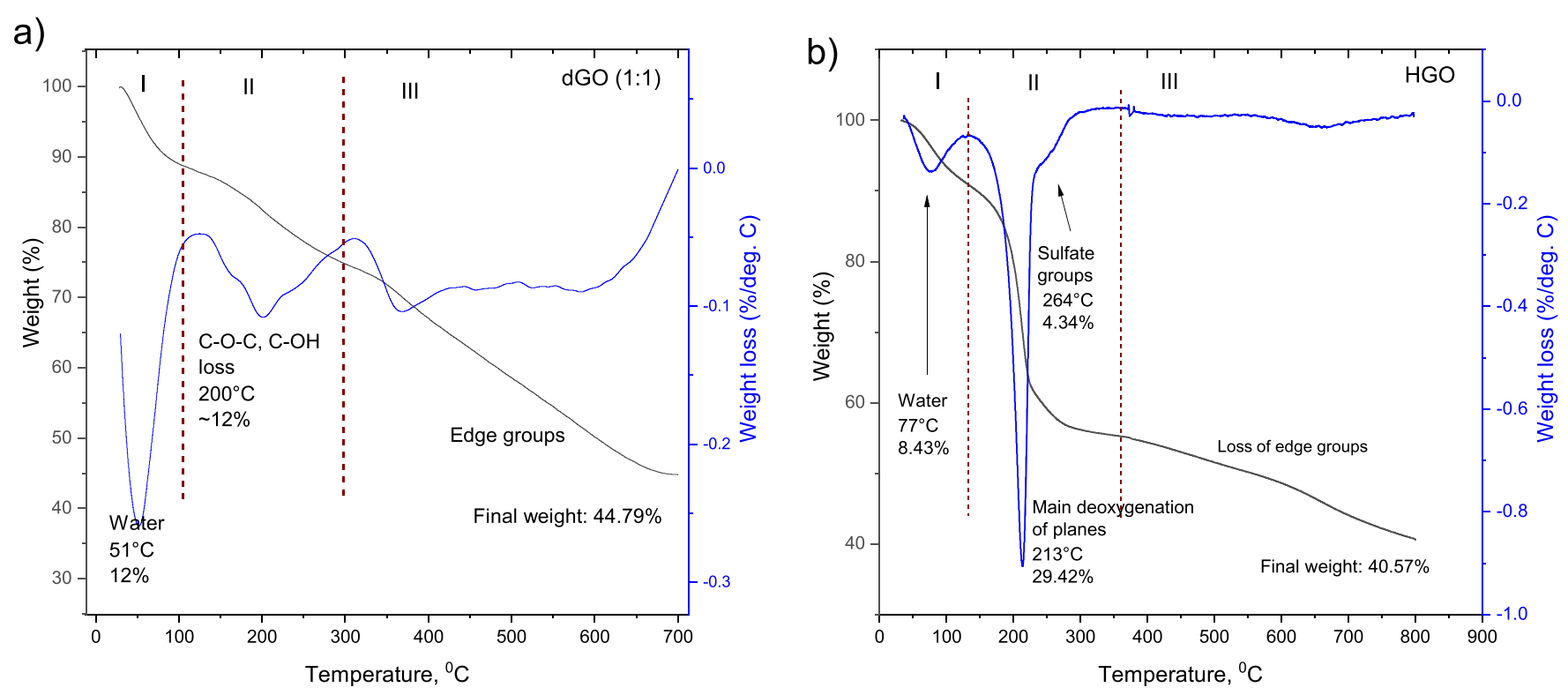

Figure 7. TGA traces of (a) dGO and (b) precursor HGO. 
which occurs in interlayers of standard HGO, does not happen in $\mathrm{dGO}$.

The defect structure is also in agreement with the FTIR spectrum of dGO shown in Figure 8. The spectra were

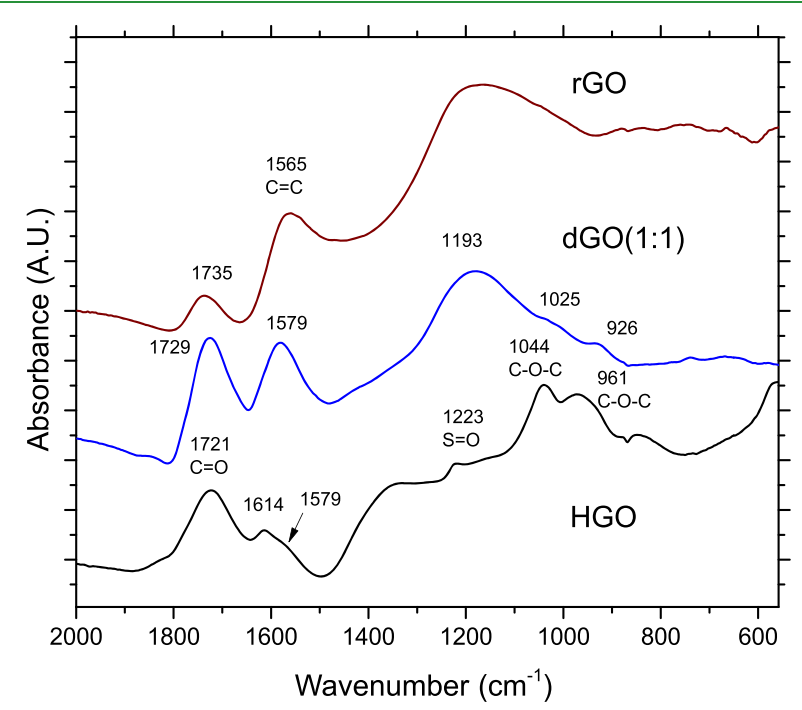

Figure 8. FTIR spectra of HGO, rGO produced by explosive exfoliation of this HGO, and dGO (1:1) prepared by Hummers oxidation of rGO. Spectra recorded using ATR under vacuum conditions.

recorded in vacuum conditions to minimize the amount of adsorbed water. The spectrum of precursor GO shows a peak from the $\mathrm{C}=\mathrm{C}$ of the graphene skeleton at $1565 \mathrm{~cm}^{-1}$ and peaks from $\mathrm{C}=\mathrm{O}$ at $1735 \mathrm{~cm}^{-1}$. The oxygen in $\mathrm{rGO}$ is mostly bound to edge carbon atoms, but some oxygen groups on the planar surface of sheets are also not completely removed by thermal reduction. It is known that complete removal of oxygen from rGO is not observed even after heating at very high temperatures. The relatively mild exfoliation temperature used in our study results in $\mathrm{C} / \mathrm{O}=5-6$. Oxidation of $\mathrm{rGO}$ is reflected in FTIR spectra by the strong increase of the $\mathrm{C}=\mathrm{O}$ peak, which also downshifts to $1729 \mathrm{~cm}^{-1}$, more similar to the position of the $\mathrm{C}=\mathrm{O}$ peak in HGO. The position of the $\mathrm{C}=\mathrm{C}$ peak shifted from $1565 \mathrm{~cm}^{-1}$ in rGO to $1579 \mathrm{~cm}^{-1}$ of dGO (1:1), also more similar to standard HGO. However, the FTIR spectra provide clear evidence that Hummers oxidation of $\mathrm{rGO}$ results in the synthesis of a material rather different from HGO.

The peaks typically assigned to $\mathrm{C}-\mathrm{O}-\mathrm{C}$ and $\mathrm{C}-\mathrm{OH}$ are rather weak in the spectra of $\mathrm{dGO}$ reflecting the extremely defect nature of this material and the absence of a sufficient planar surface for these functional groups. In fact, the spectrum of dGO exhibits some features similar to rGO and some features similar to HGO in FTIR spectra. It is known that the choice of the precursor carbon material strongly affects the properties of GO.

Sorption Properties of dGO. Sorption of Methylene Blue. It is known that GO shows a high sorption capacity for methylene blue (MB). Moreover, sorption of $\mathrm{MB}$ was used in several studies for the evaluation of surface area accessible for GO in aqueous solutions. ${ }^{16,17}$ Therefore, we performed a rapid test to verify our initial assumption that the defect structure improves sorption properties of dGO. Indeed, the dGO sorption of methylene blue was found to be significantly enhanced compared to that of standard HGO. As a reference, we tested also sorption of MB by BGO powder. As expected from our earlier studies, the more defect nature of HGO than BGO results in a twice higher amount of adsorbed MB (428 and $184 \mathrm{mg} / \mathrm{g}$, respectively). The sorption of $\mathrm{MB}$ by dGO is $879 \mathrm{mg} / \mathrm{g}$, more than twice higher than that of standard HGO. Graphite oxides are known to exhibit a rather small surface area by gas sorption but significant surface area in water solutions. ${ }^{17}$ The difference is explained by the effect of swelling. The nitrogen gas molecules do not penetrate between GO layers, but the interlayer distance expands significantly due to intercalation of liquid water. Swelling of the GO structure provides the possibility for dissolved molecules to enter interlayers of $\mathrm{GO}$ and to be adsorbed in significant amounts.

The gravimetric amounts of adsorbed $\mathrm{MB}$ are often recalculated into effective surface area values, as shown in Table 2. ${ }^{16,62}$ Using this method, dGO shows an MB sorption

Table 2. BET Surface Areas Determined Using Analysis of Nitrogen Sorption Isotherms, Saturated Sorption of MB by HGO, BGO, and dGO (1:1), and Effective Surface Areas ${ }^{a}$

$\begin{array}{cccc}\text { sample } & \begin{array}{c}\text { BET SSA } \\ \left(\mathrm{m}^{2} / \mathrm{g}\right)\end{array} & \begin{array}{c}\text { methylene blue sorption } \\ (\mathrm{mg} / \mathrm{g} \text { active material })\end{array} & \begin{array}{c}\text { effective surface area } \\ \left(\mathrm{m}^{2} / \mathrm{g}\right)\end{array} \\ \mathrm{HGO} & <5 & 428 & 1086 \\ \mathrm{BGO} & <5 & 184 & 466 \\ \mathrm{dGO} & 19 & 879 & 2233 \\ \quad(1: 1) & & 586 & 1489 \\ \begin{array}{c}\text { porous } \\ \text { carbon }\end{array} & 2007 & & \\ \end{array}$

$a_{\text {The }} \mathrm{MB}$ sorption does not correlate with the surface area determined using nitrogen sorption isotherms. Surface area was calculated assuming $2.54 \mathrm{~m}^{2}$ per $\mathrm{mg}$ of adsorbed $\mathrm{MB}$ according to Montes-Navajas et al.

surface area about $50 \%$ higher than the reference sample of porous carbon with a BET $\left(\mathrm{N}_{2}\right)$ of $\sim 2000 \mathrm{~m}^{2 /} \mathrm{g}$. However, our results indicate that the estimation of the surface area using MB needs to be treated with caution as it is obviously dependent on the amount of defects in GO. Both HGO and BGO swell well, thus providing a very similar surface area in liquid water, but the sorption of $\mathrm{MB}$ is rather different (Table $2)$. The difference in the defect state is also likely to be a reason for rather strong spread in reported gravimetric values for $\mathrm{MB}$ sorption by $\mathrm{GO}$ in the literature, typically 250-450 $\mathrm{mg} / \mathrm{g}^{62,63}$ but with some reports up to $\sim 700 \mathrm{mg} / \mathrm{g}{ }^{64}$

Sorption of Radionuclides. Sorption of $\mathrm{U}(\mathrm{VI})$ and $\mathrm{Am}(\mathrm{III}) / \mathrm{Eu}(\mathrm{III})$ was tested for dGO and standard HGO (Figures 9 and 10). It is known that $\mathrm{U}(\mathrm{VI})$ is present in solution in the form of uranyl cation $\mathrm{UO}_{2}{ }^{2+}$ with a total charge of +2 and effective charge of +3.2 . The Am(III) cation has a charge of $+3 .^{65}$ In agreement with our expectations, dGO (1:1) demonstrated a higher sorption ability for both $\mathrm{U}(\mathrm{VI})$ and $\mathrm{Am}$ (III) over the entire range of $\mathrm{pH}$ (Figure 9). The largest difference in sorption is observed in very low $\mathrm{pH}$ conditions. The $\mathrm{pH}$ value at $50 \%$ of sorption $(\mathrm{pH} 50)$ is smaller for $\mathrm{dGO}(1: 1)$ by 1.1 unit in the case of $\mathrm{U}(\mathrm{VI})$, while for Am(III), it is 0.65 unit.

The enhanced sorption properties of $\mathrm{dGO}$ are attributed to the higher concentration of carboxyl groups compared to those of HGO. Therefore, the sorption isotherm of radionuclides was used to estimate the amount of functional groups. The isotherms were first fitted by the Langmuir model: $C_{\text {sorb }}=$ $Q_{\max } \cdot K_{\mathrm{La}} \cdot C_{\mathrm{sol}} /\left(1+K_{\mathrm{La}} C_{\text {sol }}\right)$, where $C_{\text {sorb }}$ is the equilibrium 

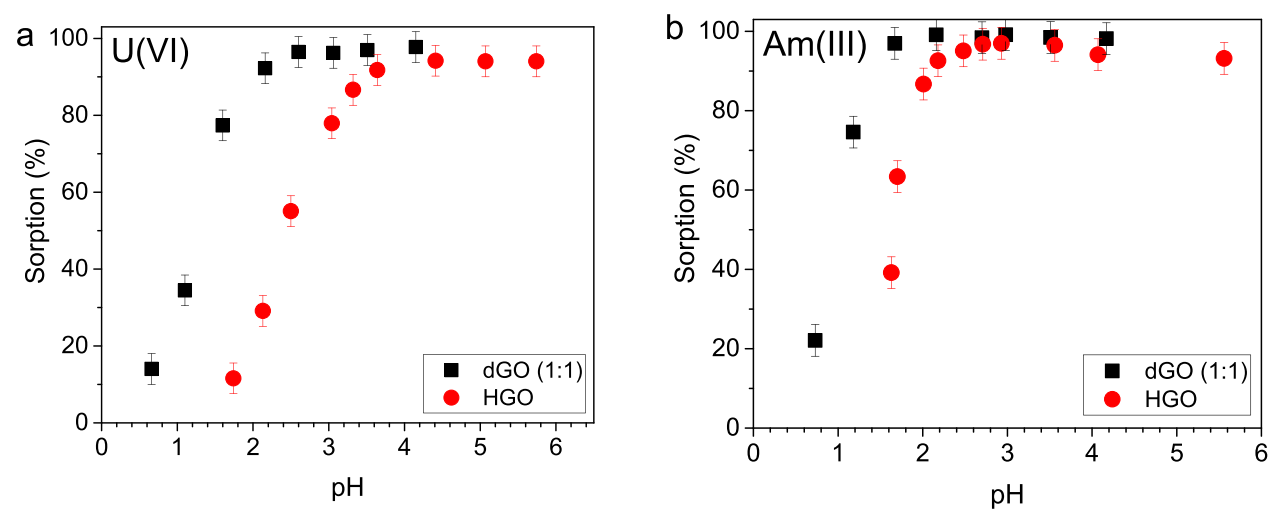

Figure 9. $\mathrm{pH}$ sorption edges for (a) $\mathrm{U}(\mathrm{VI})$ and (b) Am(III) onto dGO (1:1) and initial HGO ([Am(III) $\left.]=6 \times 10^{-10} \mathrm{M},[\mathrm{U}(\mathrm{VI})]=2 \times 10^{-7} \mathrm{M}\right)$.
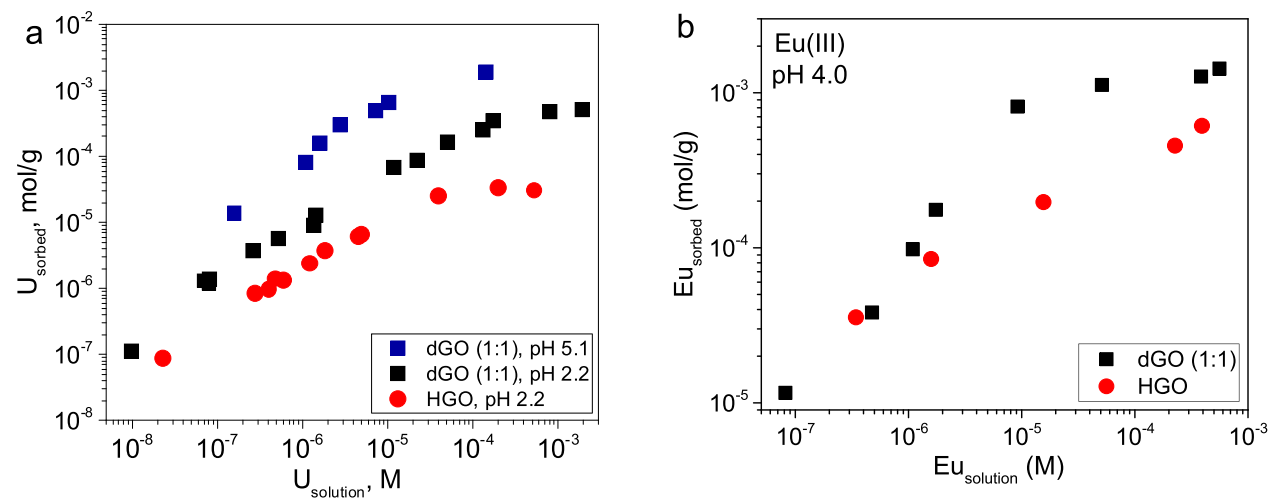

Figure 10. Sorption isotherms of radionuclides (a) U(VI) and (b) Eu(III) onto HGO and dGO (1:1).

concentration of adsorbed radionuclides, $C_{\text {sol }}$ is the equilibrium concentration of radionuclides in aqueous solution, $Q_{\max }$ is the maximum sorption capacity, and $K_{\mathrm{La}}$ is a constant. According to the fit of experimental data (Table 3 ), the sorption capacity

Table 3. Sorption Capacity of the Studied Samples Calculated by Applying the Langmuir Model

\begin{tabular}{lcc} 
& \multicolumn{2}{c}{ maximum sorption capacity $Q_{\max } \mu \mathrm{mol} / \mathrm{g}$} \\
\cline { 2 - 3 } sample & $\mathrm{U}(\mathrm{VI})$ at $\mathrm{pH} 2.2$ & $\mathrm{Eu}(\mathrm{III})$ at $\mathrm{pH} 4.0$ \\
$\mathrm{HGO}$ & $35 \pm 1$ & $597 \pm 58$ \\
$\mathrm{dGO}(1: 1)$ & $551 \pm 12$ & $1367 \pm 50$
\end{tabular}

of dGO is significantly higher than that of $\mathrm{HGO}-$ ca. 15 times for $\mathrm{U}(\mathrm{VI})$ and ca. 2 times for Am(III). As it was discussed above, dGO has a higher amount of carboxylic groups than pristine $\mathrm{HGO}$, in agreement with our previous results where interaction of radionuclides with carboxyl groups proved to be a main mechanism of interaction.

Comparing the sorption capacities determined from the Langmuir formalism for different experimental conditions is hardly possible. There are a lot of factors that influence sorption capacity, most importantly the $\mathrm{pH}$ value and cation type. In particular, the $\mathrm{pH}$ value of the sorption isotherm has a drastic effect on sorption capacity of GO as demonstrated also in our earlier studies. ${ }^{2}$ There is no uniform standard for reporting sorption at certain $\mathrm{pH}$ in the literature. Nevertheless, we compared the sorption capacities of the studied material determined from the sorption isotherms of dGO with the literature data (Table 4) for several common sorbent materials. dGO demonstrates extremely high sorption toward U(VI), significantly exceeding sorption capacity of CNTs, AC, and standard GO.

Table 4. Comparing of Sorption Capacities from Literature Reference and this Study

\begin{tabular}{llcc}
$\quad$ sorbent & $\begin{array}{c}\mathrm{pH} \\
\text { value }\end{array}$ & $\begin{array}{c}\text { maximum sorption capacity } \\
Q_{\text {max }} \mu \mathrm{mol} / \mathrm{g}\end{array}$ & ref \\
$\begin{array}{l}\text { natural zeolite } \\
\text { clinoptilolite }\end{array}$ & & 5.2 & 68 \\
$\begin{array}{l}\text { nanozeolite } \\
\text { nanozeolite/ZnO } \\
\text { composite }\end{array}$ & 7.56 & 12 & 69 \\
biochar & 7.56 & 23 & \\
GO & 5.5 & 114 & 70 \\
activated carbon & 5 & 116 & 2,3 \\
carbon nanotubes & 5 & 119 & 71 \\
modified carbon & 5 & 166 & 3,72 \\
$\quad$ nanotubes & 5.5 & 193 & 73 \\
native algal biomass & 5.5 & 828 & 74 \\
modified algal biomass & 5.5 & 1510 & 74 \\
dGO (1:1) & 5.1 & 2250 & this \\
& & & work \\
\hline
\end{tabular}

A more sophisticated approach to describe sorption reaction is surface complexation modeling (SCM), assuming the sorption process as an exchange with a proton in the following reaction: $\equiv \mathrm{SOH}+\mathrm{Cat}^{n+} \rightleftarrows \equiv \mathrm{SOCat}^{(n-1)+}+\mathrm{H}^{+}$, where $\equiv$ $\mathrm{SOH}$ is the functional group on the surface, in particular case the carboxyl group.

This concept has found wide application for describing sorption of cations by different minerals. ${ }^{66,67}$ This approach was applied also to GO systems by some authors. ${ }^{21,23,24,26}$ 
However, even in the case of standard GO, this approach can be quite controversial due to the variety of functional groups found in GO. At least 5-7 oxygen functional groups are described in a standard structural model of $\mathrm{GO},{ }^{44}$ making modeling difficult and ambiguous.

Moreover, the concentrations of functional groups in other studies $^{21,23,24,26}$ were determined by potentiometric titration. However, because of the complex structure of GO, not all functional groups that interact with $\mathrm{H}^{+}$may interact with other cations.

In the present study, dGO samples contain predominantly carboxyl groups. Therefore, we performed SCM assuming that interaction occurs with a single averaged type of sorption site. This assumption also supports the sorption isotherm shape where only one plateau is reached. In addition, unlike other works, we performed simultaneous fitting of all sorption data for $\mathrm{Am}(\mathrm{III}) / \mathrm{Eu}(\mathrm{III}$ ) and $\mathrm{U}(\mathrm{VI})$ (two $\mathrm{pH}$-curves and two isotherms). A nonelectrostatic model (NEM) was used for the modeling assuming that sorption occurs inside the hole defects on the graphene planes. We tested both formation of monoand poly-dentate complexes onto the dGO surface. Better results were found using the concept of monodentate complex formation (Table 5).

Table 5. Parameters of Optimization Sorption Data Using the SCM Approach

\begin{tabular}{|c|c|}
\hline & $\log K$ \\
\hline$\equiv \mathrm{SOH} \rightleftarrows \equiv \mathrm{SO}^{-}+\mathrm{H}^{+}$ & $-4^{a}$ \\
\hline$\equiv \mathrm{SOH}+\mathrm{UO}_{2}^{2+} \rightleftarrows \equiv \mathrm{SOUO}_{2}^{+}+\mathrm{H}^{+}$ & 2.35 \\
\hline$\equiv \mathrm{SOH}+\mathrm{Am}^{3+} \rightleftarrows \equiv \mathrm{SOAm}^{2+}+\mathrm{H}^{+}$ & 3.60 \\
\hline$\equiv \mathrm{SOH}+\mathrm{Eu}^{3+} \rightleftarrows \equiv \mathrm{SOEu}^{2+}+\mathrm{H}^{+}$ & 2.20 \\
\hline$[\equiv \mathrm{SOH}], \mathrm{mol} / \mathrm{g}$ & $7 \times 10^{-4}$ \\
\hline
\end{tabular}

The fit of the experimental data is adequate (Figure S2). The concentration of surface carboxyl groups was calculated to be 7 $\times 10^{-4} \mathrm{~mol} / \mathrm{g}$, that is, 3.3 times higher than for standard GO in Xie and Powell's work. ${ }^{25}$ This result confirms that the defect nature of re-oxidized rGO with an increased number of carboxylic groups is favorable for sorption. The interaction of radionuclides with GO occurs through complexation with carboxyl groups. Therefore, the linear free energy relationship (LFER) between constants of interaction of radionuclides with simple carboxylic acids and GO should take place. In the present work, we found that the sorption constants of Am(III), $\mathrm{Eu}(\mathrm{III})$, and $\mathrm{U}(\mathrm{VI})$ onto $\mathrm{dGO}$ are in a perfect linear correlation with the complexation constants with the acetate ion (Figure S3).

To clarify the mechanism of radionuclide sorption by dGO, we recorded EXAFS spectra after equilibration with cations. Analysis of the EXAFS spectra allows some conclusions to be made on the local atomic environment of sorbed uranium.

Fitted experimental EXAFS spectra of U(VI) adsorbed by dGO (1:1) are presented in Figure 11 with corresponding structural parameters listed in Table 6. The EXAFS spectrum is rather similar to that for $\mathrm{U}(\mathrm{VI})$ on reference HGO suggesting a similar mechanism of sorption. ${ }^{1}$ In the $\mathrm{U}(\mathrm{VI})$ spectrum, the first peak of the Fourier transform at $1.3-1.4 \AA$ is the U-O shell and can be fitted with two axial uranyl oxygen atoms in the $\mathrm{UO}_{2}{ }^{2+}$ cation. The next peak corresponds to equatorial oxygen atoms and is fitted by two subshells with resulting coordination numbers of 2.26 and 2.41. The latter subshell corresponds to $\mathrm{U}-\mathrm{C}$ interactions. Analysis of EXAFS spectra demonstrates that the dGO material sorbs $\mathrm{U}(\mathrm{VI})$ with the exact same mechanism as the reference HGO, with carboxylic groups dominating the $\mathrm{GO}-$ cation interaction.

Additional evidence for the suggested mechanism of U(VI) sorption by dGO was obtained using high-resolution transmission electron microscopy (HRTEM) (Figure 12). Heavy atoms provide higher contrast in HRTEM and are relatively easy for imaging. However, images of the $\mathrm{dGO}$ sample with sorbed U(VI) demonstrate the absence of clustering, formation of nanoparticles, or extra concentration of uranium
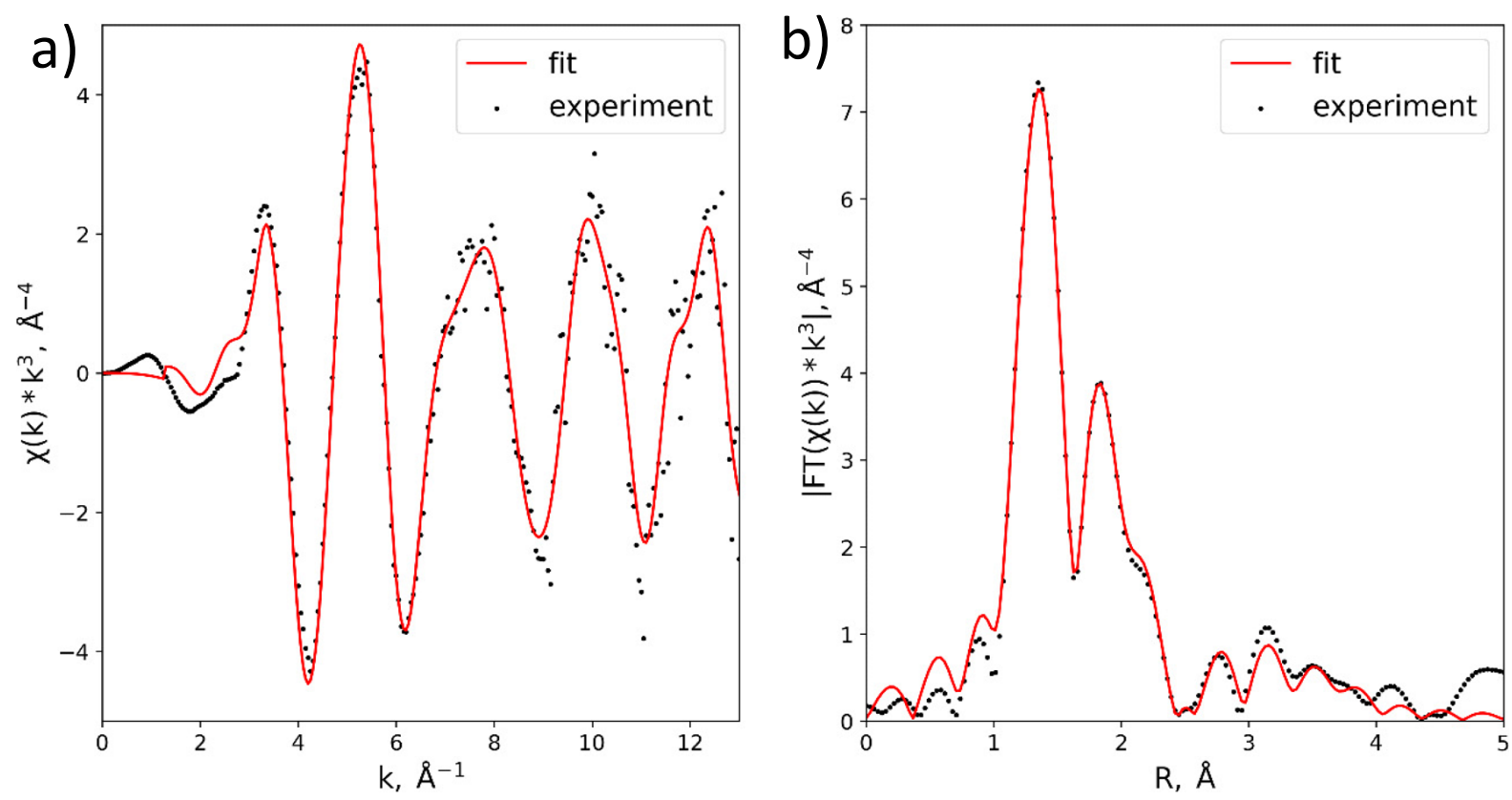

Figure 11. EXAFS spectra of $\mathrm{U}(\mathrm{VI})$ sorbed onto $\mathrm{dGO}(1: 1)$ : (a) oscillation part of the EXAFS spectra, (b) absolute values of Fourier transformations of the spectra. Dots represent the experimental data; lines are the fitting of the spectra. 
Table 6. Structural Parameters around Uranium and GO Samples Derived from EXAFS Analyses ${ }^{a}$

\begin{tabular}{|c|c|c|c|c|c|c|}
\hline sample & & $\mathrm{CN}$ & $R, \AA$ & $\sigma, \AA^{2}$ & $\Delta E, \mathrm{eV}$ & \\
\hline \multirow[t]{6}{*}{$\mathrm{U}(\mathrm{VI})+\mathrm{dGO}(1: 1)$} & $\mathrm{U}-\mathrm{O}$ & $2.0^{*}$ & 1.77 & 0.002 & 6.2 & $R$-factor $=0.005, R$-range $=1.1-4.0 \AA, k$-range $=3.0-13.0 \AA^{-1}$ \\
\hline & $\mathrm{U}-\mathrm{O}$ & 1.6 & 2.26 & 0.002 & & \\
\hline & $\mathrm{U}-\mathrm{O}$ & 4.4 & 2.41 & 0.006 & & \\
\hline & $\mathrm{U}-\mathrm{C}$ & 1.7 & 2.94 & 0.003 & & \\
\hline & $\mathrm{U}-\mathrm{C}$ & 4.8 & 3.81 & 0.003 & & \\
\hline & $\mathrm{U}-\mathrm{C}$ & 4.5 & 4.00 & 0.003 & & \\
\hline \multirow[t]{7}{*}{$\mathrm{U}(\mathrm{VI})+\mathrm{HGO}$, ref 1} & $\mathrm{U}-\mathrm{O}$ & $2.0 *$ & 1.75 & 0.001 & 2.2 & $R$-factor $=0.002$ \\
\hline & $\mathrm{U}-\mathrm{O}$ & 2.3 & 2.22 & 0.002 & & \\
\hline & $\mathrm{U}-\mathrm{O}$ & 4.1 & 2.39 & 0.003 & & \\
\hline & $\mathrm{U}-\mathrm{C}$ & 0.6 & 2.68 & 0.002 & & \\
\hline & $\mathrm{U}-\mathrm{C}$ & 2.6 & 2.91 & 0.002 & & \\
\hline & $\mathrm{U}-\mathrm{C}$ & 4.1 & 3.76 & 0.003 & & \\
\hline & $\mathrm{U}-\mathrm{C}$ & 3.7 & 3.95 & 0.003 & & \\
\hline
\end{tabular}

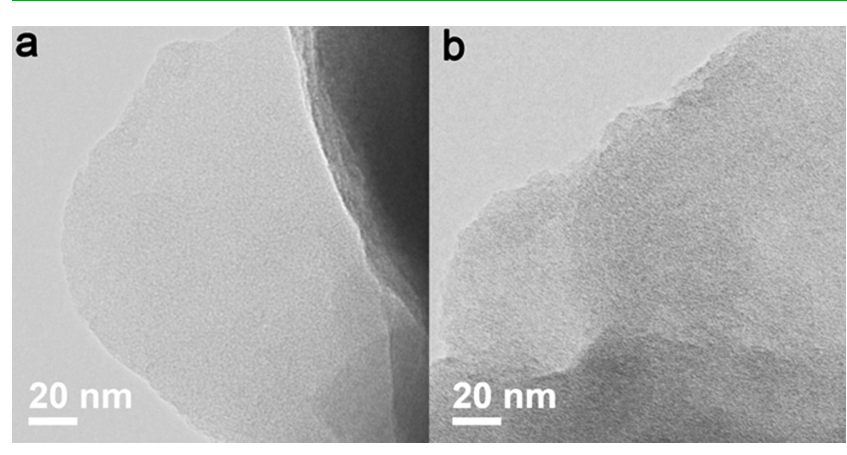

Figure 12. HRTEM images recorded from (a) precursor dGO (1:1) and (b) dGO(1:1) with adsorbed U(VI).

along the edges of flakes. In the STEM regime, the images also show that uranium is distributed homogeneously over the whole surface of dGO flakes. (Figure S4). The data suggest that $\mathrm{U}(\mathrm{VI})$ is bound to structural defects in GO sheets, which are rather small in size, below the resolution of the images and homogeneously distributed over the whole area of flakes. According to our previous results based on EXAFS and modeling, the main sorption sites in HGO for the binding of radionuclides are carboxylic groups on the inside edges of small $\sim 1 \mathrm{~nm}$-sized holes. ${ }^{1}$ HRTEM images are in agreement with the suggested mechanism, showing a homogeneous distribution of uranium over the entire surface of dGO flakes.

Further confirmation of the similarity of the sorption mechanisms in dGO and standard HGO comes from HERFD-XANES measured at the Uranium $\mathrm{M}_{4}$ edge, which probes directly the f-orbitals through $3 \mathrm{~d}_{3 / 2} \rightarrow 5 \mathrm{f}_{5 / 2}$ electronic transitions. The spectra measured on $\mathrm{U}(\mathrm{VI})$ sorbed onto HGO and dGO are shown in Figure 13. The spectra show the characteristic profile of the uranyl ion, with three wellseparated peaks, which have been assigned to transitions to the nonbonding $5 \mathrm{f} \delta_{\mathrm{u}}$ and $5 \mathrm{f} \varphi_{\mathrm{u}}$, antibonding $5 \mathrm{f} \pi_{\mathrm{u}}{ }^{*}$, and antibonding $5 \mathrm{f} \sigma_{\mathrm{u}}{ }^{*}$ molecular orbitals. ${ }^{75}$

Aside from a small decrease in the intensity of the second peak in dGO compared with $\mathrm{HGO}$, the spectra are almost identical, indicative of a very similar local coordination environment in the two materials. In particular, the fact that the third peak is unchanged in energy position is a strong indication that the short $\mathrm{U}-\mathrm{O}$ axial bonds are identical. Indeed, as $\mathrm{M}_{4}$ HERFD probes directly the antibonding

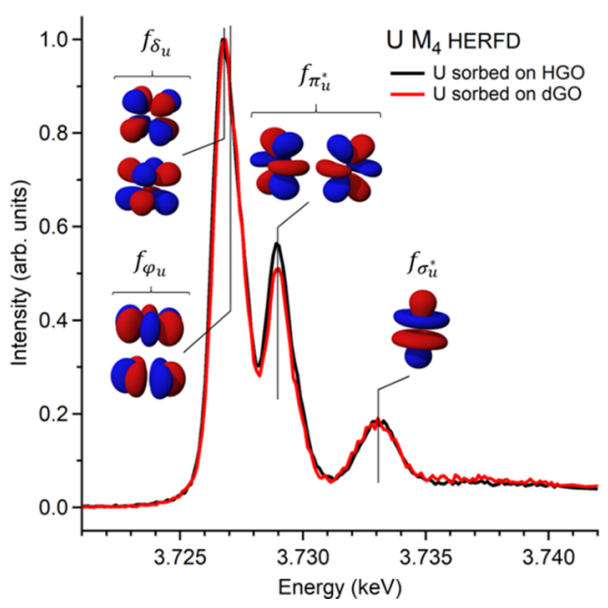

Figure 13. $\mathrm{U} \mathrm{M}_{4}$ edge HERFD spectra of $\mathrm{U}(\mathrm{VI})$ sorbed on $\mathrm{HGO}$ and dGO (1:1). The three peaks correspond to transitions to the unoccupied molecular orbitals with $\mathrm{U} f$ character. Drawings of the $\mathrm{f}$ orbitals involved in each transition are shown according to Vitova et al. $^{75}$

molecular orbitals of uranyl, this measurement is extremely sensitive to structural changes.

Previous $\mathrm{M}_{4}$ HERFD measurements of uranyl in lithium borate glasses show that a $0.06 \AA$ change of the bond length can result in a shift of the third peak $(\mathrm{f} \sigma)$ by as much as $1 \mathrm{eV}{ }^{76}$ A small shift can be recognized in the HERFD-XANES spectra of $\mathrm{U}(\mathrm{VI})$ sorbed onto GO synthesized using three different methods: Hummers, Tour, and Brodie (Figure S5), thus confirming the high sensitivity of this method to elongation of bond length. These three types of GO were characterized in detail in our previous study. ${ }^{1}$ A small 0.01 A elongation of the uranyl bond was found for the BGO material using EXAFS, ${ }^{1}$ and indeed, the third peak in the $\mathrm{M}_{4}$ edge spectrum shows a shift of $0.7 \mathrm{eV}$ to lower energy. An increase of the $\mathrm{U}-\mathrm{O}$ axial bond distance results in energetic stabilization of the $5 \mathrm{f} \sigma_{\mathrm{u}}{ }^{*}$ orbital, which, based on multiplet calculations (Figure S6), shifts the third peak to lower energies, in agreement with the experimental observations. The small intensity decrease of the second peak in dGO spectra indicates some differences in the local environment of $\mathrm{U}(\mathrm{VI})$. However, the effect is minor and might be related to the equatorial $\mathrm{U}-\mathrm{O}$ bonds, whose effect on the local electronic structure is weak. 
Summarizing the data presented above, the mechanism of uranyl sorption by dGO is essentially the same as in standard HGO, which has a higher number of defects than BGO. This result confirms that the material obtained by Hummers oxidation of rGO is also graphene oxide with all major properties typical for standard HGO but with a significant increase in the number of defects, which provides the 15 -fold increase of uranyl sorption. It is a nontrivial result considering that the oxidation of defect-rich rGO is likely to result in the formation of a variety of defects, e.g., holes of different diameters, edges of different shapes, etc. A relatively large overall number of carboxylic groups around the flake edges are also expected due to the small flake size of dGO. However, uranyl cations are sorbed on dGO by a mechanism remarkably similar to standard HGO. The spectra in Figures 11 and 13 show no new spectral features, which could indicate the appearance of abundant sites with a different mechanism of sorption. No increased concentration of uranium is also found in HRTEM images on the edges of GO flakes (Figure 12).

Therefore, we suggest that only very specific sorption sites related to carboxylic groups on small holes in GO sheets (as in standard $\mathrm{HGO}^{1}$ ) are responsible for most of the uranyl sorption. Many other defect types, which are inevitably formed in the process of dGO synthesis, do not contribute to the sorption of uranyl and remain unoccupied. This interpretation of data is confirmed by the fact that the number of carboxyl groups determined using XPS is about 10 times higher than the molar concentration of sorbed uranium cations. Therefore, only about $10 \%$ of carboxylic groups are providing sorption sites for uranyl, while the rest are in defects or flake edges that remain not suitable for uranyl sorption.

\section{CONCLUSIONS}

In conclusion, extremely defect-rich GO was prepared using Hummers oxidation of explosively produced defect rGO and tested for sorption of several radionuclides. In agreement with our earlier fundamental study of radionuclides binding to different types of GO, carboxylic groups located on the edges of small holes are responsible for the sorption of, e.g., uranyl cations. The dGO material was therefore designed specifically with the aim to increase the number of defects. Precursor rGO was intentionally prepared using explosive exfoliation in conditions that provide the maximal number of defects. Thermal reduction of GO is known to proceed with the evolvement of gaseous carbon oxides by removing carbon atoms from the graphene skeleton and producing abundant vacancy defects and holes. Further oxidation of highly dispersed and defect $\mathrm{rGO}$ results in the formation of a material that has about 10 -fold higher number of carboxylic groups than standard HGO and an unusually low number of oxygen groups, which are typically found on the planar surface of GO sheets. A simple analysis of XPS data allows estimation that about $10 \%$ of all carbon atoms in dGO are located on the edges of flakes or inner edges of holes, thus forming an extremely defect structure.

In agreement with our expectation, the dGO material showed a 15-fold increase in sorption of U(VI) and 2-fold increase in sorption of Am(III). As revealed by high-resolution microscopy, the uranyl cations are homogeneously distributed over the dGO flakes, showing no aggregation. Analysis of electron and synchrotron-based spectroscopy data - EXAFS and HERFD-XANES - allows concluding that the enhanced sorption of uranyl by dGO is related to the same type of defect as in standard HGO. According to modeling results, uranyl cations are bound to carboxylic groups located inside the small holes in dGO and HGO structures. However, the overall number of these defects is higher in dGO, which results in a significantly improved sorption capacity, which can be useful in many applications related to the treatment of toxic and radioactive wastes.

\section{ASSOCIATED CONTENT}

\section{Supporting Information}

The Supporting Information is available free of charge at https://pubs.acs.org/doi/10.1021/acsami.0c11122.

$\mathrm{N}_{2}$ sorption isotherms for dGO and thermally exfoliated rdGO materials, figures presenting modeling of sorption data, STEM images of dGO with sorbed U(VI), HERFD-XANES and EXAFS recorded for U(VI) sorbed onto $\mathrm{HGO}$, TGO, and $\mathrm{BGO}$, theoretical $\mathrm{U} \mathrm{M}_{4}$ HERFD, and additional XPS spectra of precursor materials (PDF)

\section{AUTHOR INFORMATION}

\section{Corresponding Authors}

Stepan N. Kalmykov - Department of Chemistry, Lomonosov Moscow State University, Moscow 119991, Russia;

Email: stepan@radio.chem.msu.ru

Alexandr V. Talyzin - Department of Physics, Umeå University, S-90187 Umeå, Sweden; 이이.org/0000-0002-3320-8487; Email: alexandr.talyzin@umu.se

\section{Authors}

Nicolas Boulanger - Department of Physics, Umeå University, S-90187 Umeä, Sweden

Anastasiia S. Kuzenkova - Department of Chemistry, Lomonosov Moscow State University, Moscow 119991, Russia

Artem Iakunkov - Department of Physics, Umea University, S90187 Umeå, Sweden

Anna Yu. Romanchuk - Department of Chemistry, Lomonosov Moscow State University, Moscow 119991, Russia

Alexander L. Trigub - Department of Chemistry, Lomonosov Moscow State University, Moscow 119991, Russia; National Research Centre "Kurchatov Institute", Moscow 123098, Russia

Alexander V. Egorov - Department of Chemistry, Lomonosov Moscow State University, Moscow 119991, Russia

Stephen Bauters - The Rossendorf Beamline at ESRF, The European Synchrotron, 38043 Grenoble, France; Helmholtz Zentrum Dresden-Rossendorf (HZDR), Institute of Resource Ecology, 01314 Dresden, Germany; 이이.org/0000-00015484-8857

Lucia Amidani - The Rossendorf Beamline at ESRF, The European Synchrotron, 38043 Grenoble, France; Helmholtz Zentrum Dresden-Rossendorf (HZDR), Institute of Resource Ecology, 01314 Dresden, Germany; 이이.org/0000-00032234-4173

Marius Retegan - The European Synchrotron, 38043 Grenoble, France; orcid.org/0000-0002-1525-1094

Kristina O. Kvashnina - The Rossendorf Beamline at ESRF, The European Synchrotron, 38043 Grenoble, France; Helmholtz Zentrum Dresden-Rossendorf (HZDR), Institute of Resource Ecology, 01314 Dresden, Germany; Department of Chemistry, Lomonosov Moscow State University, Moscow 119991, Russia; (1) orcid.org/0000-0003-4447-4542

Complete contact information is available at: https://pubs.acs.org/10.1021/acsami.0c11122 


\section{Author Contributions}

$\nabla$ N.B. and A.S.K. contributed equally. The manuscript was written by contributions of all authors. A.V.T. conceived the idea for synthesis of the dGO material, XPS interpretation for characterization of the defect state, supervised the part about synthesis and characterization, and wrote relevant parts of the manuscript. N.B. performed the synthesis and characterization of the material with A.I.'s assistance. A.I. modeled the data for XPS interpretation. A.S.K. carried out radionuclide sorption experiments. A.Yu.R. supervised radionuclide sorption experiments, made modeling of sorption data, and co-wrote a draft of the manuscript. A.L.T., K.O.K. supervised EXAFS and HERFD part carried out measurements and fitting of the EXAFS spectra of $\mathrm{U}(\mathrm{VI})$ sorption onto dGO samples, and co-wrote a draft of the manuscript. A.V.E. carried out HRTEM measurements. S.N.K. supervised work, co-wrote a draft of the manuscript. S.B. performed the HERFD experiment at the $U$ $\mathrm{M}_{4}$ edge. K.O.K., L.A., and M.R analyzed the HERFD data and performed theoretical simulations. K.O.K, S.B., L.A., and M.R. co-wrote the HERFD part of the manuscript. All authors participated in discussions and preparation of the manuscript. All authors have given approval to the final version of the manuscript.

\section{Notes}

The authors declare no competing financial interest.

\section{ACKNOWLEDGMENTS}

N.B. and A.V.T. acknowledge funding from the European Union's Horizon 2020 Research and Innovation Program under grant agreement No 785219 and No 881603. Support from Swedish Research Council grant (no. 2017-04173) is also acknowledged. We also acknowledge the Vibrational Spectroscopy Platform of Umea University and A. Shchukarev for support with the XPS test. The experimental studies were partially performed on equipment acquired from funding by the Lomonosov Moscow State University Development Program. Authors acknowledge help of J. Rothe, K. Dardenne, A. Beck, T. Prussman, and T. Vitova at the CAT-ACT beamline of KARA during the HERFD-XANES experiment at the $\mathrm{U} \mathrm{M}_{4}$ edge. We would like to thank $S$. Weiss from the HZDR safety group for his help in handling radioactive samples for the HERFD-XANES experiment. L.A, S.B., and K.O.K. acknowledge the support from the European Research Council (ERC) (grant agreement No 759696) (HERFDXANES experiment). A.S.K, A.L.T., and S.N.K. acknowledge support by the Russian Ministry of Science and Education under grant No 075-15-2019-1891 (sorption experiments on radionuclides).

\section{REFERENCES}

(1) Kuzenkova, A. S.; Romanchuk, A. Y.; Trigub, A. L.; Maslakov, K. I.; Egorov, A. V.; Amidani, L.; Kittrell, C.; Kvashnina, K. O.; Tour, J. M.; Talyzin, A. V.; Kalmykov, S. N. New insights into the mechanism of graphene oxide and radionuclide interaction. Carbon 2020, 158, 291-302.

(2) Romanchuk, A. Y.; Slesarev, A. S.; Kalmykov, S. N.; Kosynkin, D. V.; Tour, J. M. Graphene oxide for effective radionuclide removal. Phys. Chem. Chem. Phys. 2013, 15, 2321-2327.

(3) Romanchuk, A. Y.; Kuzenkova, A. S.; Slesarev, A. S.; Tour, J. M.; Kalmykov, S. N. Cs(I) and Sr(II) Sorption onto Graphene Oxide. Solvent Extr. Ion Exch. 2016, 34, 594-602.

(4) Borai, E. H.; Harjula, R.; Malinen, L.; Paajanen, A. Efficient removal of cesium from low-level radioactive liquid waste using natural and impregnated zeolite minerals. J. Hazard. Mater. 2009, 172, 416-422.

(5) Pansini, M. Natural zeolites as cation exchangers for environmental protection. Miner. Deposita 1996, 31, 563-575.

(6) De Windt, L.; Pellegrini, D.; van der Lee, J. Coupled modeling of cement/claystone interactions and radionuclide migration. J. Contam. Hydrol. 2004, 68, 165-182.

(7) Li, K.; Pang, X. Sorption of radionuclides by cement-based barrier materials. Cem. Concr. Res. 2014, 65, 52-57.

(8) Abdel-Karim, A. A. M.; Zaki, A. A.; Elwan, W.; El-Naggar, M. R.; Gouda, M. M. Experimental and modeling investigations of cesium and strontium adsorption onto clay of radioactive waste disposal. Appl. Clay Sci. 2016, 132-133, 391-401.

(9) Miller, A. W.; Wang, Y. Radionuclide Interaction with Clays in Dilute and Heavily Compacted Systems: A Critical Review. Environ. Sci. Technol. 2012, 46, 1981-1994.

(10) Bradbury, M. H.; Baeyens, B. Modelling the sorption of $\mathrm{Mn}(\mathrm{II}), \mathrm{Co}(\mathrm{II}), \mathrm{Ni}(\mathrm{II}), \mathrm{Zn}(\mathrm{II}), \mathrm{Cd}(\mathrm{II}), \mathrm{Eu}(\mathrm{III}), \mathrm{Am}(\mathrm{III}), \mathrm{Sn}(\mathrm{IV})$, $\mathrm{Th}(\mathrm{IV}), \mathrm{Np}(\mathrm{V})$ and $\mathrm{U}(\mathrm{VI})$ on montmorillonite: Linear free energy relationships and estimates of surface binding constants for some selected heavy metals and actinides. Geochim. Cosmochim. Acta 2005, 69, 875-892.

(11) Durrant, C. B.; Begg, J. D.; Kersting, A. B.; Zavarin, M. Cesium sorption reversibility and kinetics on illite, montmorillonite, and kaolinite. Sci. Total Environ. 2018, 610-611, 511-520.

(12) Mahmoud, M. R.; Sharaf El-Deen, G. E.; Soliman, M. A. Surfactant-impregnated activated carbon for enhanced adsorptive removal of $\mathrm{Ce}(\mathrm{IV})$ radionuclides from aqueous solutions. Ann. Nucl. Energy 2014, 72, 134-144.

(13) Wang, Y.; Gao, H.; Yeredla, R.; Xu, H.; Abrecht, M. Control of pertechnetate sorption on activated carbon by surface functional groups. J. Colloid Interface Sci. 2007, 305, 209-217.

(14) Savage, N.; Diallo, M. S. Nanomaterials and water purification: Opportunities and challenges. J. Nanopart. Res. 2005, 7, 331-342.

(15) Mackeyev, Y. A.; Marks, J. W.; Rosenblum, M. G.; Wilson, L. J. Stable containment of radionuclides on the nanoscale by cut singlewall carbon nanotubes. J. Phys. Chem. B 2005, 109, 5482-5484.

(16) Montes-Navajas, P.; Asenjo, N. G.; Santamaría, R.; Menéndez, R.; Corma, A.; García, H. Surface Area Measurement of Graphene Oxide in Aqueous Solutions. Langmuir 2013, 29, 13443-13448.

(17) Boehm, H. P.; Clauss, A.; Fischer, G.; Hofmann, C. Surface Properties of Extremely Thin Graphite Lamellae. Proc. 5th Conf. on Carbon (Oxford: Pergamon); Pergamon Press, 1962, 73-80.

(18) Sun, J.; Morales-Lara, F.; Klechikov, A.; Talyzin, A. V.; Baburin, I. A.; Seifert, G.; Cardano, F.; Baldrighi, M.; Frasconi, M.; Giordani, S. Porous Graphite Oxide Pillared with Tetrapod-Shaped Molecules. Carbon 2017, 120, 145-156.

(19) Tan, L.; Wang, S.; Du, W.; Hu, T. Effect of water chemistries on adsorption of Cs(I) onto graphene oxide investigated by batch and modeling techniques. Chem. Eng. J. 2016, 292, 92-97.

(20) Wang, C. L.; Li, Y.; Liu, C. L. Sorption of uranium from aqueous solutions with graphene oxide. J. Radioanal. Nucl. Chem. 2015, 304, 1017-1025.

(21) Xie, Y.; Helvenston, E. M.; Shuller-Nickles, L. C.; Powell, B. A. Surface Complexation Modeling of $\mathrm{Eu}(\mathrm{III})$ and $\mathrm{U}(\mathrm{VI})$ Interactions with Graphene Oxide. Environ. Sci. Technol. 2016, 50, 1821-1827.

(22) Li, Z.; Chen, F.; Yuan, L.; Liu, Y.; Zhao, Y.; Chai, Z.; Shi, W. Uranium(VI) adsorption on graphene oxide nanosheets from aqueous solutions. Chem. Eng. J. 2012, 210, 539-546.

(23) Duster, T. A.; Szymanowski, J. E. S.; Fein, J. B. Experimental Measurements and Surface Complexation Modeling of U(VI) Adsorption onto Multilayered Graphene Oxide: The Importance of Adsorbate-Adsorbent Ratios. Environ. Sci. Technol. 2017, 51, 85108518.

(24) Gu, D.; Fein, J. B. Adsorption of metals onto graphene oxide: Surface complexation modeling and linear free energy relationships. Colloids Surf., A 2015, 481, 319-327. 
(25) Xie, Y.; Powell, B. A. Linear Free Energy Relationship for Actinide Sorption to Graphene Oxide. ACS Appl. Mater. Interface 2018, 10, 32086-32092.

(26) Hu, T.; Ding, S.; Deng, H. Application of three surface complexation models on $\mathrm{U}(\mathrm{VI})$ adsorption onto graphene oxide. Chem. Eng. J. 2016, 289, 270-276.

(27) Amirov, R. R.; Shayimova, J.; Nasirova, Z.; Dimiev, A. M. Chemistry of Graphene Oxide. Reactions with Transition Metal Cations. Carbon 2017, 116, 356-365.

(28) Hummers, W. S., Jr.; Offeman, R. E. Preparation of Graphitic Oxide. J. Am. Chem. Soc. 1958, 80, 1339-1339.

(29) Brodie, B. C. XIII. On the Atomic Weight of Graphite. Philos. Trans. R. Soc. London 1859, 149, 249-259.

(30) Marcano, D. C.; Kosynkin, D. V.; Berlin, J. M.; Sinitskii, A.; Sun, Z.; Slesarev, A.; Alemany, L. B.; Lu, W.; Tour, J. M. Improved Synthesis of Graphene Oxide. ACS Nano 2010, 4, 4806-4814.

(31) Boehm, H. P.; Scholz, W. Der Verpuffungspunkt Des Graphitoxids. Z. Anorg. Allg. Chem. 1965, 335, 74-79.

(32) You, S.; Luzan, S. M.; Szabó, T.; Talyzin, A. V. Effect of Synthesis Method on Solvation and Exfoliation of Graphite Oxide. Carbon 2013, 52, 171-180.

(33) Talyzin, A. V.; Mercier, G.; Klechikov, A.; Hedenström, M.; Johnels, D.; Wei, D.; Cotton, D.; Opitz, A.; Moons, E. Brodie vs Hummers Graphite Oxides for Preparation of Multi-Layered Materials. Carbon 2017, 115, 430-440.

(34) Talyzin, A. V.; Klechikov, A.; Korobov, M.; Rebrikova, A. T.; Avramenko, N. V.; Gholami, M. F.; Severin, N.; Rabe, J. P. Delamination of Graphite Oxide in a Liquid upon Cooling. Nanoscale 2015, 7, 12625-12630.

(35) Talyzin, A. V.; Hausmaninger, T.; You, S.; Szabó, T. The Structure of Graphene Oxide Membranes in Liquid Water, Ethanol and Water-Ethanol Mixtures. Nanoscale 2014, 6, 272-281.

(36) You, S.; Luzan, S.; Yu, J.; Sundqvist, B.; Talyzin, A. V. Phase Transitions in Graphite Oxide Solvates at Temperatures Near Ambient. J. Phys. Chem. Lett. 2012, 3, 812-817.

(37) Feicht, P.; Siegel, R.; Thurn, H.; Neubauer, J. W.; Seuss, M.; Szabó, T.; Talyzin, A. V.; Halbig, C. E.; Eigler, S.; Kunz, D. A.; Fery, A.; Papastavrou, G.; Senker, J.; Breu, J. Systematic Evaluation of Different Types of Graphene Oxide in Respect to Variations in Their In-Plane Modulus. Carbon 2017, 114, 700-705.

(38) Korobov, M. V.; Talyzin, A. V.; Rebrikova, A. T.; Shilayeva, E. A.; Avramenko, N. V.; Gagarin, A. N.; Ferapontov, N. B. Sorption of Polar Organic Solvents and Water by Graphite Oxide: Thermodynamic Approach. Carbon 2016, 102, 297-303.

(39) Szabó, T.; Berkesi, O.; Forgó, P.; Josepovits, K.; Sanakis, Y.; Petridis, D.; Dékány, I. Evolution of Surface Functional Groups in a Series of Progressively Oxidized Graphite Oxides. Chem. Mater. 2006, $18,2740-2749$.

(40) Krishnamoorthy, K.; Veerapandian, M.; Yun, K.; Kim, S. J. The Chemical and Structural Analysis of Graphene Oxide with Different Degrees of Oxidation. Carbon 2013, 53, 38-49.

(41) Dimiev, A. M.; Alemany, L. B.; Tour, J. M. Graphene Oxide. Origin of Acidity, Its Instability in Water, and a New Dynamic Structural Model. ACS Nano 2013, 7, 576-588.

(42) Kim, S.; Zhou, S.; Hu, Y.; Acik, M.; Chabal, Y. J.; Berger, C.; de Heer, W.; Bongiorno, A.; Riedo, E. Room-Temperature Metastability of Multilayer Graphene Oxide Films. Nat. Mater. 2012, 11, 544-549.

(43) Iakunkov, A.; Sun, J.; Rebrikova, A.; Korobov, M.; Klechikov, A.; Vorobiev, A.; Boulanger, N.; Talyzin, A. V. Swelling of Graphene Oxide Membranes in Alcohols: Effects of Molecule Size and Air Ageing. J. Mater. Chem. A 2019, 7, 11331-11337.

(44) Lerf, A.; He, H.; Forster, M.; Klinowski, J. Structure of Graphite Oxide Revisited. J. Phys. Chem. B 1998, 102, 4477-4482.

(45) Erickson, K.; Erni, R.; Lee, Z.; Alem, N.; Gannett, W.; Zettl, A. Determination of the Local Chemical Structure of Graphene Oxide and Reduced Graphene Oxide. Adv. Mater. 2010, 22, 4467-4472.

(46) Liu, Z.; Nørgaard, K.; Overgaard, M. H.; Ceccato, M.; Mackenzie, D. M. A.; Stenger, N.; Stipp, S. L. S.; Hassenkam, T.
Direct Observation of Oxygen Configuration on Individual Graphene Oxide Sheets. Carbon 2018, 127, 141-148.

(47) Gogoi, A.; Koneru, A.; Anki Reddy, K. Effect of Graphene Oxide (GO) Nanosheet Sizes, Pinhole Defects and Non-Ideal Lamellar Stacking on the Performance of Layered GO Membranes: An Atomistic Investigation. Nanoscale Adv. 2019, 1, 3023-3035.

(48) Feicht, P.; Eigler, S. Defects in Graphene Oxide as Structural Motifs. Chemnanomat 2018, 4, 244-252.

(49) Kaewmee, P.; Manyam, J.; Opaprakasit, P.; Le, G. T. T.; Chanlek, N.; Sreearunothai, P. Effective Removal of Cesium by Pristine Graphene Oxide: Performance, Characterizations and Mechanisms. RSC Adv. 2017, 7, 38747-38756.

(50) Amirov, R. R.; Shayimova, J.; Nasirova, Z.; Solodov, A.; Dimiev, A. M. Analysis of Competitive Binding of Several Metal Cations by Graphene Oxide Reveals the Quantity and Spatial Distribution of Carboxyl Groups on its Surface. Phys. Chem. Chem. Phys. 2018, 20, 2320-2329.

(51) Cheng, M.; Yang, R.; Zhang, L.; Shi, Z.; Yang, W.; Wang, D.; Xie, G.; Shi, D.; Zhang, G. Restoration of Graphene from Graphene Oxide by Defect Repair. Carbon 2012, 50, 2581-2587.

(52) Eigler, S.; Dotzer, C.; Hirsch, A. Visualization of Defect Densities in Reduced Graphene Oxide. Carbon 2012, 50, 3666-3673.

(53) Overgaard, M. H.; Kühnel, M.; Hvidsten, R.; Petersen, S. V.; Vosch, T.; Nørgaard, K.; Laursen, B. W. Highly Conductive Semitransparent Graphene Circuits Screen-Printed from WaterBased Graphene Oxide Ink. Adv. Mater. Technol. 2017, 2, 1700011.

(54) Klechikov, A. G.; Mercier, G.; Merino, P.; Blanco, S.; Merino, C.; Talyzin, A. V. Hydrogen Storage in Bulk Graphene-Related Materials. Microporous Mesoporous Mater. 2015, 210, 46-51.

(55) Talyzin, A. V.; Szabó, T.; Dékány, I.; Langenhorst, F.; Sokolov, P. S.; Solozhenko, V. L. Nanocarbons by High-Temperature Decomposition of Graphite Oxide at Various Pressures. J. Phys. Chem. C 2009, 113, 11279-11284.

(56) Newville, M. EXAFS analysis using FEFF and FEFFIT. J. Synchrotron Radiat. 2001, 8, 96-100.

(57) Zimina, A.; Dardenne, K.; Denecke, M. A.; Doronkin, D. E.; Huttel, E.; Lichtenberg, H.; Mangold, S.; Pruessmann, T.; Rothe, J.; Spangenberg, T.; Steininger, R.; Vitova, T.; Geckeis, H.; Grunwaldt, J. D. CAT-ACT-A new highly versatile $\mathrm{x}$-ray spectroscopy beamline for catalysis and radionuclide science at the KIT synchrotron light facility ANKA. Rev Sci Instrum 2017, 88, 113113.

(58) Rothe, J.; Altmaier, M.; Dagan, R.; Dardenne, K.; Fellhauer, D.; Gaona, X.; Corrales, E. G. R.; Herm, M.; Kvashnina, K. O.; Metz, V.; Pidchenko, I.; Schild, D.; Vitova, T.; Geckeis, H. Fifteen Years of Radionuclide Research at the KIT Synchrotron Source in the Context of the Nuclear Waste Disposal Safety Case. Geosciences 2019, 9, 91.

(59) Baburin, I. A.; Klechikov, A.; Mercier, G.; Talyzin, A.; Seifert, G. Hydrogen Adsorption by Perforated Graphene. Int. J. Hydrogen Energy 2015, 40, 6594-6599.

(60) Klechikov, A.; Sun, J.; Hu, G.; Zheng, M.; Wågberg, T.; Talyzin, A. V. Graphene Decorated with Metal Nanoparticles: Hydrogen Sorption and Related Artefacts. Microporous Mesoporous Mater. 2017, 250, 27-34.

(61) McAllister, M. J.; Li, J. L.; Adamson, D. H.; Schniepp, H. C.; Abdala, A. A.; Liu, J.; Herrera-Alonso, M.; Milius, D. L.; Car, R.; Prud'homme, R. K.; Aksay, I. A. Single Sheet Functionalized Graphene by Oxidation and Thermal Expansion of Graphite. Chem. Mater. 2007, 19, 4396-4404.

(62) Boehm, H. P.; Clauss, A.; Fischer, G. O.; Hofmann, U. Das Adsorptionsverhalten Sehr Dunner Kohlenstoff-Folien. Z. Anorg. Allg. Chem. 1962, 316, 119-127.

(63) Bradder, P.; Ling, S. K.; Wang, S.; Liu, S. Dye Adsorption on Layered Graphite Oxide. J. Chem. Eng. Data 2011, 56, 138-141.

(64) Yang, S.-T.; Chen, S.; Chang, Y.; Cao, A.; Liu, Y.; Wang, H. Removal of Methylene Blue from Aqueous Solution by Graphene Oxide. J Colloid Interface Sci 2011, 359, 24-29.

(65) Choppin, G. R. Actinide speciation in aquatic systems. Mar. Chem. 2006, 99, 83-92. 
(66) Brendler, V.; Vahle, A.; Arnold, T.; Bernhard, G.; Fanghänel, T. (REST)-T-3-Rossendorf expert system for surface and sorption thermodynamics. J. Contam. Hydrol. 2003, 61, 281-291.

(67) Payne, T. E.; Brendler, V.; Ochs, M.; Baeyens, B.; Brown, P. L.; Davis, J. A.; Ekberg, C.; Kulik, D. A.; Lutzenkirchen, J.; Missana, T.; Tachi, Y.; Van Loon, L. R.; Altmann, S. Guidelines for thermodynamic sorption modelling in the context of radioactive waste disposal. Environ Modell. Software 2013, 42, 143-156.

(68) Kilincarslan, A.; Akyil, S. Uranium adsorption characteristic and thermodynamic behavior of clinoptilolite zeolite. J. Radioanal. Nucl. Chem. 2005, 264, 541-548.

(69) Shakur, H. R.; Saraee, K. R. E.; Abdi, M. R.; Azimi, G. Selective Removal of Uranium Ions from Contaminated Waters using Modified-X Nanozeolite. Appl. Radiat. Isot. 2016, 118, 43-55.

(70) Mishra, V.; Sureshkumar, M. K.; Gupta, N.; Kaushik, C. P. Study on Sorption Characteristics of Uranium onto Biochar Derived from Eucalyptus Wood. Water Air Soil Pollut. 2017, 228, 309.

(71) Mellah, A.; Chegrouche, S.; Barkat, M. The removal of uranium(VI) from aqueous solutions onto activated carbon: Kinetic and thermodynamic investigations. J. Colloid Interface Sci. 2006, 296, 434-441.

(72) Fasfous, I. I.; Dawoud, J. N. Uranium (VI) sorption by multiwalled carbon nanotubes from aqueous solution. Appl. Surf. Sci. 2012, 259, 433-440.

(73) Schierz, A.; Zänker, H. Aqueous suspensions of carbon nanotubes: Surface oxidation, colloidal stability and uranium sorption. Environ. Pollut. 2009, 157, 1088-1094.

(74) Bayramoglu, G.; Akbulut, A.; Acıkgoz-Erkaya, I.; Yakup Arica, M. Uranium sorption by native and nitrilotriacetate-modified Bangia atropurpurea biomass: kinetics and thermodynamics. J. Appl. Phycol. 2018, 30, 649-661.

(75) Vitova, T.; Green, J. C.; Denning, R. G.; Löble, M.; Kvashnina, K.; Kas, J. J.; Jorissen, K.; Rehr, J. J.; Malcherek, T.; Denecke, M. A. Polarization Dependent High Energy Resolution X-ray Absorption Study of Dicesium Uranyl Tetrachloride. Inorg. Chem. 2015, 54, 174182.

(76) Hunault, M. O. J. Y.; Lelong, G.; Cormier, L.; Galoisy, L.; Solari, P. L.; Calas, G. Speciation Change of Uranyl in Lithium Borate Glasses. Inorg. Chem. 2019, 58, 6858-6865. 